\title{
Vanishing viscosity limits of mixed hyperbolic-elliptic systems arising in multilayer channel flows
}

\author{
E.S. Papaefthymiou and D.T. Papageorgiou \\ Department of Mathematics, Imperial College London, London SW7 2AZ, UK
}

\begin{abstract}
.
This study considers the spatially periodic initial value problem of $2 \times 2$ quasi-linear parabolic systems having quadratic polynomial flux functions. These systems arise physically in the interfacial dynamics of viscous immiscible multilayer channel flows. The equations describe the spatiotemporal evolution of phase-separating interfaces with dissipation arising from surface tension (fourth order) and/or stable stratification effects (second order). A crucial mathematical aspect of these systems is the presence of mixed hyperbolic-elliptic flux functions that provide the only source of instability. The study concentrates on scaled spatially $2 \pi-$ periodic solutions as the dissipation vanishes, and in particular the behaviour of such limits when generalised dissipation operators (spanning second to fourth order) are considered. Extensive numerical computations and asymptotic analysis suggest that the existence (or not) of bounded vanishing viscosity solutions depends crucially on the structure of the flux function. In the absence of linear terms (i.e. homogeneous flux functions) the vanishing viscosity limit does not exist in the $\mathrm{L}^{\infty}-$ norm. On the other hand, if linear terms in the flux function are present the computations strongly suggest that the solutions exist and are bounded in the $\mathrm{L}^{\infty}$ - norm as the dissipation vanishes. It is found that the key mechanism that provides such boundedness centres on persistent spatiotemporal hyperbolic-elliptic transitions. Strikingly, as the dissipation decreases, the flux function becomes almost everywhere hyperbolic except on a fractal set of elliptic regions, whose dimension depends on the order of the regularized operator. Furthermore, the spatial structures of the emerging weak solutions are found to support an increasing number of discontinuities (measure-valued solutions) located in the vicinity of the fractally distributed elliptic regions. For the unscaled problem, such spatially oscillatory solutions can be realized as extensive dynamics analogous to those found in the Kuramoto-Sivashinsky equation.
\end{abstract}




\section{Introduction}

Many problems arising from fluid physics are governed by nonlinear partial differential equations (PDEs) that are systems of conservation laws of mixed hyperbolic-elliptic type. Particular examples can be found in stratified flows (see [1, 2], for example), steady transonic flows [3], magneto-fluid dynamics [4], sedimentation of polydisperse suspensions [5], fluids of van der Waals type [6], three-phase flow in porous media modeling fluid flows in petroleum reservoirs [7], as well as in viscoelastic fluids [8]. Due to the presence of ellipticity (the system possesses complex eigenvalues), such nonlinear systems do not share the same kind of well-posedness as hyperbolic conservation. In the absence of any kind of regularization, the corresponding linearized system is catastrophically unstable in the sense of Hadamard - linear theory predicts that short waves grow the fastest, causing problems for numerical computations (see [9] and [10], for example). Furthermore, sufficient criteria for admissible solutions of mixed-type systems are an open problem since their vanishing viscosity limits, if they exist, are sensitive to the structure of regularization. Consequently, the practical and mathematical meaningfulness of the mixed hyperbolic-elliptic models have been questioned and the existence of a complete theory, as in the case of hyperbolic conservation laws, is a debatable issue (see Keyfitz [11], for a discussion). One of the main objectives of this work is to explore and understand the behaviour of general vanishing viscosity limits of mixed hyperbolic-elliptic systems that possess quadratic nonlinearities. In this paper we consider a class of mixed-type $2 \times 2$ conservation laws that are derived physically (from first principles) and describe the interfacial dynamics of stratified multilayer flows $[12]$.

In the scalar case, such as the Burgers canonical nonlinear hyperbolic equation, the existence and the uniqueness of the global Cauchy problem is completely known (see [13]); in addition, admissibility criteria, specially designed to select physically relevant weak solutions, coincide with each other. However, the main existence and uniqueness theorems for general $N \times N$ systems of conservation laws demand additional restrictions to be imposed. In a fundamental paper, Glimm [14] proved existence of global weak entropy solutions provided that the $N \times N$ quasilinear system is strictly hyperbolic, each characteristic field is either genuinely nonlinear or linearly degenerate in the sense of Lax [15], and the initial data are of small total variation. For $N=2$, Glimm \& Lax [16] have weakened the condition on the initial data, allowing small oscillations. However, this stronger result cannot be extended to the case $N \geq 3$, since nonlinear resonance mechanisms can produce unbounded amplifications and as a result even strictly hyperbolic systems can blow up in finite time (see [17] and the references therein). For an extensive review on the basic theory of one-dimensional strictly hyperbolic systems of conservation laws and a description of the remaining open problems see [18]. In a celebrated paper, Bianchini \& Bressan [19] used the vanishing viscosity method to prove global existence and uniqueness of general $N \times N$ strictly hyperbolic systems, requiring only small total variations on the initial data. 
They considered diffusion limits (i.e. second-order dissipation) with the matrix of the regularized term being the identity matrix. The concept of general viscosity matrices is an open mathematical problem. Majda \& Pego [20] identified stable viscosity matrices for systems of hyperbolic conservation laws. Furthermore, even in the scalar case one cannot expect global uniqueness for generalized vanishing viscosity limits. This can be illustrated by the following two special examples; the zero diffusion limit of the Burgers equation and the zero dispersion limit of the Kortweg de-Vries equation. In the former case the regularized system converges strongly to the weak solution in the limit, while in the latter case, oscillations persist ([21] and references therein). Bertozzi et al. [22] studied fourth-order regularized scalar conservation laws describing the interfacial dynamics of thin film flows. They show that in contrast to second order dissipation, fourth order regularization alters the behaviour of the corresponding inviscid conservation law, admitting non-classical shock waves in the limit of vanishing viscosity. Furthermore, the lack of monoticity in the case of fourth-order regularization implies nontrivial linear stability of the non-uniform viscous profiles, [23]. Finally, we would like to mention that the multi-dimensional problem of systems of hyperbolic conservation laws is terra incognita and their mathematical development is highly desirable. For instance, the existence or not of singular solutions at large times for three-dimensional (3D) incompressible Euler equations is a long-standing open problem and of great significance since it can provide an avenue towards the mechanism for the energy transfer to small scales in turbulence, [24].

The Cauchy problem of mixed hyperbolic-elliptic systems is a subject of considerable interest and is under analytical investigation by several research teams. The main tool utilised is the vanishing viscosity method, where one seeks to extract a subsequence of a regularized solutions $\left\{\mathbf{u}^{(\epsilon)}\right\}$ which converge to a weak solution of the associated inviscid system as the viscosity $\epsilon$ tends to zero. Uniform controls on the amplitude (the spatial norm) and derivative (total variation norm) of the approximate solutions $\left\{\mathbf{u}^{(\epsilon)}\right\}$ can provide a compactness argument and, consequently, one can show that a subsequence will converge strongly to $\mathbf{u}$. This is exactly the case for strictly hyperbolic systems of conservation laws reviewed previously. However, in the case of mixed-type systems, estimates on the total variation are not generally available, and one can find uniform $\mathrm{L}^{\infty}$ bounds only for special systems; this is possible in cases where bounded invariant regions exist as identified by Chueh et al. [25]. Such a priori bounds can only guarantee weak convergence, that is convergence in the local averages (in the weak-* topology of $\mathrm{L}^{\infty}$ ). The failure to show strong convergence emanates from the fact that the approximate regularized solutions may not converge to a weak solution but are found to develop rapid oscillations as $\epsilon$ approaches zero. By using the method of compensated compactness, DiPerna [21] introduced the notion of measurevalued solutions which generalize the concept of the weak solutions and provide a framework for the study of the persistence of oscillations in the vanishing viscosity limit. Admissible measure-valued solutions of the systems endowed with positively invariant regions reduce to a Dirac solution, suggesting that oscillations are not present 
in the limit and there exists a subsequence of the approximate solutions that converge strongly to a weak solution inside these regions (see [26] for the case of isentropic gas dynamics). On the other hand, if this is not the case and oscillations persist in the limit, the approximate regularized solutions converge to a measure-valued solution which corresponds to a non-classical weak solution (with non-Dirac structure). Consequently, the structure of the measure-valued solution reflects the character of the convergence and the type of oscillations that are present in the vanishing viscosity limit (see DiPerna [21]). DiPerna \& Majda [27] extended the notion of the measure-valued solutions to the context of $\mathrm{L}^{p}$ function sequences in order to deal with complex dynamical behavior of oscillations and/or concentrations developed in the limit of approximate solutions to the 3D incompressible Euler equations.

Finally, when the Riemann problem is considered for mixed-type conservation laws, one should expect the presence of oscillations and non-classical weak solutions in the vanishing viscosity limit. Peters \& Canic [28] used numerical solutions to show that the vanishing viscosity method for the Riemann problem of the shallow water wave equations yields limits which exhibit persistent and stable oscillations that increase in frequency but stay uniformly bounded as the viscosity term vanishes, and proved that this limit is a measure-valued solution of the system. Furthermore, Frid \& Liu [29] studied numerically Riemann problems for mixed-type systems with initial data inside elliptic regions, and observed non-classical (measure valued) solutions with persistent oscillations. Berres et al. [30] captured such oscillations in models of sedimentation of polydisperse suspensions by employing multiresolution schemes.

In this paper, we deal with general vanishing viscosity limits of $2 \times 2$ mixed hyperbolic-elliptic systems which incorporate quadratic polynomial flux functions. We consider the initial value problem with spatially periodic boundary conditions and mostly random initial conditions. The randomness of the initial conditions ensures that the flux function of the system initially supports both hyperbolic and elliptic regions - hence we exclude special cases encountered in the literature that initialise the problem inside invariant hyperbolic regions. Intuitively, one expects that the persistence of flux function ellipticity in the vanishing viscosity limit would prevent the existence of bounded solutions, and such behaviour is confirmed for homogeneous quadratic flux functions. However, our study also suggests that this is not the case in general. By probing the spatiotemporal evolution of the elliptic regions in the case of inhomogeneous flux functions we found that such nonlinear structures give rise to persistent hyperbolicelliptic transitions. Interestingly, these transitions enable the existence of bounded but highly oscillatory solutions in the limit (these are measure-valued solutions). Moreover, as the viscosity vanishes, the elliptic regions are confined to a fractal Cantor-like set. Finally, we would like to emphasize that the fractal dimension associated with the emerging measure-valued solutions, depends on the order of the dissipation operator. This observation may provide an avenue towards the classification of measure-valued solutions according to the structure of the regularizing operator as has been proposed by DiPerna $[21,31]$. 
The structure of the rest of the paper is as follows. Section 2 provides the description of the mathematical problem along with a derivation of the system of equations to be studied here. Section 3 presents numerical solutions as the viscosity decreases for homogeneous flux functions, while section 4 considers analogous results for the inhomogeneous case. Finally, in section 5 some concluding remarks are made along with a short discussion of a future work.

\section{Description of the problem}

Consider the following $2 \times 2$ system in one-space dimension

$$
\frac{\partial \mathbf{U}}{\partial t}+\frac{\partial \mathbf{F}(\mathbf{U} ; \mathbf{T})}{\partial x}+\delta\left[\mathbf{G}(\mathbf{U} ; \mathbf{T}) \frac{\partial \mathbf{U}}{\partial x}+\mathbf{D}(\mathbf{U} ; \mathbf{T}) \frac{\partial^{3} \mathbf{U}}{\partial x^{3}}\right]_{x}=0
$$

subject to $L$-periodic boundary conditions, i.e. $\mathbf{U}(x+L, t)=\mathbf{U}(x, t)$, where $\mathbf{U}=$ $\left(U_{1}, U_{2}\right)^{\top} \in \mathbb{R}^{2}$ is a vector-valued function which depends on $(\mathrm{x}, \mathrm{t}) \in\left(\mathbb{R}, \mathbb{R}^{+}\right)$(the superscript $T$ denotes transpose). The $2 \times 1$ matrix $\mathbf{F}$ and the $2 \times 2$ matrices $\mathbf{G}$ and $\mathbf{D}$ have entries that are nonlinear functions of $\mathbf{U}$ and the constant 6-dimensional vector $\mathbf{T}$. System (1) is a coupled set of fully nonlinear partial differential equations (of Benneytype, [32]) that contains a small slenderness parameter $\delta \ll 1$ that cannot be scaled out of the problem. Such complex systems have been derived in the context of stratified three-layer channel flows (see [12]), where $\mathbf{U}$ represents the position of the two interfaces and $\mathbf{T}$ is the vector of the physical parameters of the problem (i.e. undisturbed interface positions, viscosity and density ratios). System (1) is valid for interfacial deformations that are long compared with typical undisturbed layer thicknesses. Furthermore, the physical origin of the order $\delta$ terms correspond to inertia and density stratification for the term proportional to $\mathbf{G}$ and surface tension for the term proportional to $\mathbf{D}$.

We proceed by performing a weakly nonlinear analysis and a Taylor expansion about fixed states $\mathbf{U}^{*} \neq 0$ and $\mathbf{T}^{*} \neq 0$ that correspond to undisturbed interface solutions. Writing $\mathbf{U}=\mathbf{U}^{*}+\delta \mathbf{u}$ and $\mathbf{T}=\mathbf{T}^{*}+\delta \mathbf{Z}$ and retaining up to order $\delta$ yields

$$
\begin{aligned}
& \frac{\partial \mathbf{u}}{\partial t}+\nabla_{\mathbf{U}} \mathbf{F}\left(\mathbf{T}^{*}\right) \frac{\partial \mathbf{u}}{\partial x}+\delta\left\{\mathbf{Z}^{\top} \nabla_{\mathbf{U T}}^{2} \mathbf{F}\left(\mathbf{T}^{*}\right) \frac{\partial \mathbf{u}}{\partial x}+\left[\frac{1}{2} \mathbf{u}^{\top} \nabla_{\mathbf{U} \mathbf{U}}^{2} \mathbf{F}\left(\mathbf{T}^{*}\right) \mathbf{u}\right]_{x}\right. \\
& \left.+\mathbf{G}\left(\mathbf{T}^{*}\right) \frac{\partial^{2} \mathbf{u}}{\partial x^{2}}+\mathbf{D}\left(\mathbf{T}^{*}\right) \frac{\partial^{4} \mathbf{u}}{\partial x^{4}}\right\}=0,
\end{aligned}
$$

where the operator $\nabla_{\mathbf{X}}$ is the Jacobian with respect to $\mathbf{X}$. The vector $\mathbf{Z}$ represents a set of six detuning parameters away from the base-states. Note that the matrices $\mathbf{G}\left(\mathbf{T}^{*}\right)$ and $\mathbf{D}\left(\mathbf{T}^{*}\right)$ have constant entries, and one can study two distinct physical scenarios regarding their values: First, inertia and density stratification absent leading to $\mathbf{G}\left(\mathbf{T}^{*}\right)=0$ and a fourth order dissipation system (the matrix $\mathbf{D}\left(\mathbf{T}^{*}\right)$ is always positive definite from physical considerations), and second, surface tension forces absent with $\mathbf{D}\left(\mathbf{T}^{*}\right)=0$ (but $\mathbf{G}\left(\mathbf{T}^{*}\right)$ a negative definite matrix, i.e. stably stratified inertialess systems), yielding second order dissipation systems. In this work we study both physical scenarios, and consequently we rewrite system (2) as follows:

$$
\frac{\partial \mathbf{u}}{\partial t}+\nabla_{\mathbf{U}} \mathbf{F}\left(\mathbf{T}^{*}\right) \frac{\partial \mathbf{u}}{\partial x}+\delta\left\{\mathbf{Z}^{\top} \nabla_{\mathbf{U T}}^{2} \mathbf{F}\left(\mathbf{T}^{*}\right) \frac{\partial \mathbf{u}}{\partial x}+\left[\frac{1}{2} \mathbf{u}^{\top} \nabla_{\mathbf{U} \mathbf{U}}^{2} \mathbf{F}\left(\mathbf{T}^{*}\right) \mathbf{u}\right]_{x}+\mathcal{L}\left(\mathbf{u} ; \mathbf{T}^{*}\right)\right\}=0
$$


where the linear operator $\mathcal{L}\left(\mathbf{u} ; \mathbf{T}^{*}\right)$ can introduce either fourth or second order dissipation as mentioned above. In the former case $\mathcal{L}\left(\mathbf{u} ; \mathbf{T}^{*}\right)=\mathbf{D}\left(\mathbf{T}^{*}\right) \frac{\partial^{4} \mathbf{u}}{\partial x^{4}}$, while in the latter it is $\mathcal{L}\left(\mathbf{u} ; \mathbf{T}^{*}\right)=\mathbf{G}\left(\mathbf{T}^{*}\right) \frac{\partial^{2} \mathbf{u}}{\partial x^{2}}$.

In order to scale out the slenderness parameter $\delta$ from system (3), we require that

$$
\nabla_{\mathbf{U}} \mathbf{F}\left(\mathbf{T}^{*}\right)=\lambda \mathbf{I} \quad \lambda \in \mathbb{R},
$$

where $\mathbf{I}$ is the identity matrix (physically this condition constrains the undisturbed states whose nonlinear stability we are investigating). This condition allows us to perform a Galilean transformation $\xi=x-\lambda t$ and remove the leading order advective terms. Finally, introduction of a new slow time-scale $\tau=\delta t$ yields the following canonical system of nonlinear coupled evolution equations

$$
\begin{aligned}
& \frac{\partial \mathbf{u}}{\partial \tau}+\zeta \mathbf{A}\left(\mathbf{T}^{*} ; \mathbf{Z}\right) \frac{\partial \mathbf{u}}{\partial \xi}+\frac{\partial \mathbf{Q}\left(\mathbf{u} ; \mathbf{T}^{*}\right)}{\partial \xi}+\mathcal{L}\left(\mathbf{u} ; \mathbf{T}^{*}\right)=0 \\
& \mathbf{u}(\xi+L, \tau)=\mathbf{u}(\xi, \tau)
\end{aligned}
$$

where the nonlinear term

$$
\mathbf{Q}:=\frac{1}{2} \mathbf{u}^{\top} \nabla_{\mathbf{U U}}^{2} \mathbf{F}\left(\mathbf{T}^{*}\right) \mathbf{u}=\left(\begin{array}{c}
a_{1}\left(\mathbf{T}^{*}\right) u_{1}^{2}+2 b_{1}\left(\mathbf{T}^{*}\right) u_{1} u_{2}+c_{1}\left(\mathbf{T}^{*}\right) u_{2}^{2} \\
c_{2}\left(\mathbf{T}^{*}\right) u_{1}^{2}+2 b_{2}\left(\mathbf{T}^{*}\right) u_{1} u_{2}+a_{2}\left(\mathbf{T}^{*}\right) u_{2}^{2}
\end{array}\right)
$$

incorporates quadratic nonlinearities. In equation $(5)$ the advective term $\zeta \mathbf{A}\left(\mathbf{T}^{*} ; \mathbf{Z}\right) \frac{\partial \mathbf{u}}{\partial \xi}$ arises from the analogous term $\mathbf{Z}^{\top} \nabla_{\mathbf{U T}}^{2} \mathbf{F}\left(\mathbf{T}^{*}\right) \frac{\partial \mathbf{u}}{\partial x}$ in (3), by introducing a single detuning parameter $\zeta$ so that $\mathbf{Z} \rightarrow \zeta \mathbf{Z}$. This enables us to manipulate the size of the advective term relative to other terms in system (5). However, retaining the six independent physical parameters $Z_{1}, \ldots, Z_{6}$, provides the freedom to control the numerical values of $2 \times 2$ matrix $\mathbf{A}$, and more importantly the nature (i.e. real or complex) of its eigenvalues.

It is useful to normalize system (5) to $2 \pi$-periodic domains by introducing new scaled coordinates

$$
x^{*}=\left(\frac{2 \pi}{L}\right) \xi, \quad t^{*}=\left(\frac{2 \pi}{L}\right) \tau,
$$

where $L$ is the spatial period of our solutions. Dropping the asterisks from independent variables yields the following system

$$
\begin{aligned}
& \frac{\partial \mathbf{u}}{\partial t}+\zeta \mathbf{A}\left(\mathbf{T}^{*} ; \mathbf{Z}\right) \frac{\partial \mathbf{u}}{\partial x}+\frac{\partial \mathbf{Q}\left(\mathbf{u} ; \mathbf{T}^{*}\right)}{\partial x}+\epsilon \mathcal{L}\left(\mathbf{u} ; \mathbf{T}^{*}\right)=0 \\
& \mathbf{u}(x+2 \pi, t)=\mathbf{u}(x, t)
\end{aligned}
$$

The resulting bifurcation parameter $\epsilon$ is given by $\epsilon=\left(\frac{2 \pi}{L}\right)^{3}>0$ when $\mathcal{L}\left(\mathbf{u} ; \mathbf{T}^{*}\right)=$ $\mathbf{D}\left(\mathbf{T}^{*}\right) \frac{\partial^{4} \mathbf{u}}{\partial x^{4}}$, whereas $\epsilon=\frac{2 \pi}{L}$ when $\mathcal{L}\left(\mathbf{u} ; \mathbf{T}^{*}\right)=\mathbf{G}\left(\mathbf{T}^{*}\right) \frac{\partial^{2} \mathbf{u}}{\partial x^{2}}$. Importantly, $\epsilon$ decreases to zero through positive values as domain size $L$ tends to infinity.

The condition (4) that enables a Galilean transformation constrains the values of $\mathbf{T}^{*}$ and determines whether the flux function (6) is hyperbolic or of mixed hyperbolicelliptic type. The hyperbolic case has been studied in detail by Schaeffer and Shearer [33] who give a classification of the kinds of hyperbolic singularities that arise depending on $\mathbf{T}^{*}$. In our problem viscosity is present and hence the hyperbolic case is of limited 
physical interest; in what follows we consider values of $\mathbf{T}^{*}$ that yield mixed-type flux functions, and we emphasize that these emerge naturally from the physical multilayer flow problem considered in [12]. Furthermore, in the present study we pick physical parameters so that the eigenvalues of $\mathbf{A}$ are complex.

The goal of the present work is the investigation of the dynamical behavior of the zero viscosity limit of the $2 \times 2$ nonlinear parabolic system (8). The existence of this limit plays an important role in the physical relevance of the reduced parabolic model. From a mathematical point of view, if one can prove that such limits exist then they represent, in the usual weak sense, solutions to the full nonlinear system (1) for which existence of global solutions is an open and challenging problem. As we have already described, the regularized operator can contain second or fourth order derivatives, so we proceed by considering a generalized diffusion operator in (8) as follows:

$$
\begin{aligned}
& \frac{\partial \mathbf{u}^{\epsilon}}{\partial t}+\zeta \mathbf{A}\left(\mathbf{T}^{*} ; \mathbf{Z}\right) \frac{\partial \mathbf{u}^{\epsilon}}{\partial x}+\frac{\partial \mathbf{Q}\left(\mathbf{u}^{\epsilon} ; \mathbf{T}^{*}\right)}{\partial x}=\epsilon \mathbf{S}\left(\mathcal{H} \circ \partial_{x}\right)^{\rho}\left[\mathbf{u}^{\epsilon}\right] \\
& \mathbf{u}(x+2 \pi, t)=\mathbf{u}(x, t)
\end{aligned}
$$

where $\rho \in[2,4], \epsilon>0$ and $\mathbf{S}$ is a constant negative definite matrix. The pseudospectral operator is defined by its Fourier transform $\mathcal{F}\left[\left(\mathcal{H} \circ \partial_{x}\right)^{\rho}[\mathbf{u}]\right](k)=|k|^{\rho} \hat{\mathbf{u}}$ and has been used in generalizations of the Kuramoto-Sivashinsky equation (see [34]) where the Hilbert transform operator $\mathcal{H}$ arises in the modeling of interfacial electrohydrodynamic problems. In addition we take $\mathbf{S}=-\mathbf{I}$, where $\mathbf{I}$ is the identity matrix, so as to avoid Majda-Pego instability, (see [20]). Note that $\rho=2$ and 4 correspond to the physical problems described by (8) since they are identical in Fourier space.

Equation (9) facilitates a mathematical study of any damping in the interval $2 \leq \rho \leq 4$ and in particular the limit $\epsilon \rightarrow 0$. Consequently we can explore numerically issues regarding the boundedness and uniqueness of the zero viscosity limit solutions. In what follows we use extensive numerical computations and asymptotic analysis to provide strong evidence for the existence (in a weak sense) of vanishing viscosity limits of (9) when $\zeta \neq 0$, as well as non-existence of global solutions for finite values of $\epsilon$ (these emerge as self-similar finite-time singularities of the PDEs) when $\zeta=0$. The crucial mechanism that facilitates existence of vanishing viscosity weak solutions when $\zeta \neq 0$, centres on the persistent spatiotemporal transitions from hyperbolic to elliptic regions and vice versa supported by the flux function (i.e. the sum of the second and third terms in (9)). Interestingly, the spatial structures of the emerging weak solutions are found to support an increasing (and unbounded) number of discontinuities that are distributed on fractal sets of dimension between 0 and 1 , with the dimension depending on the value of $\rho$. The cases $\zeta=0$ and $\zeta \neq 0$ are considered separately in the following sections. 


\section{Nonexistence of vanishing viscosity limits in the absence of linear terms in the flux function, $\zeta=0$}

In this section we investigate the nonlinear dynamical behaviour of system (9) when $\zeta=0$, i.e.

$$
\frac{\partial \mathbf{u}}{\partial t}+\frac{\partial \mathbf{Q}(\mathbf{u})}{\partial x}=-\epsilon \mathbf{I}\left(\mathcal{H} \circ \partial_{x}\right)^{\rho}[\mathbf{u}]
$$

where $\mathbf{u}=\left(u_{1}, u_{2}\right)^{\top}$. System (10) is solved on a periodic domain $x \in[-\pi, \pi]$ as the parameter $\epsilon$ decreases with the parameter $\rho$ ranging from 2 to 4 . The flux function $\mathrm{Q}$ plays a crucial role in the nonlinear dynamics and particularly the spatiotemporal evolution of its eigenvalues,

$$
\operatorname{eig}\left(\nabla_{\mathbf{u}} \mathbf{Q}\right)=\lambda_{1,2}(x, t)
$$

Our interest is in the case when the initial conditions $\mathbf{u}(x, 0)=\mathbf{u}_{o}(x)$ are such that $\lambda_{1,2}$ are not real in the whole interval, so that the inviscid system is of mixed type. This ensures that global existence theorems (for general parabolic systems) as in [35] and [25], do not apply to our system. The numerical method that we employ is a spectral method for space discretization and an implicit-explicit method for the time integration (for more details on the numerical method see [12]). Note that we can fix the initial conditions and consider $\epsilon$ decreasing to zero, or equivalently fix $\epsilon$ and increase the amplitude of the initial conditions (this follows from simple scaling of (10)). Unless otherwise stated, we will adopt the former vanishing viscosity route. In what follows we use extensive numerical computations to gain a quantitative description of the solutions in such cases.

We have identified two distinct generic cases depending on the nature of the

eigenvalues $\lambda_{1,2}$ : (a) any complex eigenvalues $\lambda_{1,2}$ are purely imaginary, and $(b)$ any complex eigenvalues have a non-zero real part. As will be shown, this difference in the nature of eigenvalues plays a crucial role in the emergence of non-trivial dynamics, which emanates purely from the coupling of the nonlinear terms. To fix matters we will present results for the following flux functions:

$$
\text { (a) } \quad \mathbf{Q}=\frac{1}{2}\left(\begin{array}{c}
u_{2}^{2} \\
u_{1}^{2}
\end{array}\right) \quad \text { and } \quad \text { (b) } \quad \mathbf{Q}=\frac{1}{2}\left(\begin{array}{c}
u_{1}^{2}+u_{2}^{2} \\
u_{1}^{2}-u_{2}^{2}
\end{array}\right) \text {. }
$$

3.1. Dynamics for fourth order dissipation $(\rho=4)$ and moderate dissipation coefficients $(\epsilon)$

We begin with a description of the dynamics for $\rho=4$ and case $(12)(a)$ with $\epsilon=1$ (sufficiently small to produce non-trivial dynamics). The initial conditions are

$$
\mathbf{u}_{o}=(9 \cos (x),-9 \cos (x))^{\top}
$$

and are selected to make $\lambda_{1,2}$ complex $\left(u_{1 o}\right.$ and $u_{2 o}$ are out-phase). Results are given in Figure 1 that shows the evolution of $u_{1}$ and $u_{2}$ (panels $(a)$ and $(b)$ ), the evolution of their respective $\mathrm{L}^{2}$-norms (panel $(c)$ ), and the space-time evolution of the nature of 
the eigenvalues (panel $(d)$ ); regions where $\lambda_{1,2}$ are complex are shaded dark and regions where they are real are white. The solution evolves to steady states that preserve the out of phase property of the initial conditions with the large time states satisfying $u_{1}(x, \cdot)=u_{2}(x+\pi, \cdot)$. Due to the symmetry of the system and the equal amplitudes of the initial conditions, the energy norms are also identical during the evolution as seen in panel $(c)$. As can be seen, the ellipticity of the eigenvalues (the whole domain supports complex eigenvalues initially) persists by forming two parallel vertical stripes in the $x-t$ plane. This is crucial in the emergence of non-trivial steady states - if the eigenvalues were to become and remain real in the whole domain, then the system is driven to the trivial state, as will be shown below. Note also that an arbitrary number of stripes (and hence spatial oscillations in the steady-state profiles) can be obtained by starting with initial conditions containing additional spatial oscillations. We illustrate this with initial conditions $\mathbf{u}_{o}=(9 \cos (8 x),-9 \cos (8 x))^{\top}$ and $\epsilon=0.001$, that lead to sixteen elliptic region stripes instead of two; the results are included in Figure $2(a, b)$ that show the evolution of the elliptic regions and the corresponding steady state profiles with the additional spatial oscillations.

We, now, consider the flux function for case $(12)(b)$ with the initial conditions given by (13), and $\epsilon=1$. Again the eigenvalues are complex in the whole domain initially, but as the results of Figure 3 show, they become real in part of the domain for relatively small times, while eventually they become real over the whole domain for $t$ larger than approximately 1.1. The system is driven to the trivial state and this is depicted in Figure $3(a)$ which shows the evolution of the $\mathrm{L}^{2}$-norms. The decay is exponential in time as expected. This change in behavior was found to be generic and depends crucially on the fact that the eigenvalues $\lambda_{1,2}$ have non-zero real parts.

For completeness we also consider case $(12)(a)$ but starting from hyperbolic initial conditions, i.e. $\lambda_{1,2}$ are real and distinct (this is achieved when $u_{1}$ and $u_{2}$ are in-phase initially). The structure of the flux function again supports non-trivial steady states at large times that emerge through a transition from hyperbolic regions (initially) to elliptic regions in the form of two vertical stripes in the $x-t$ plane as seen in Figure $4(b)$. Interestingly, the energy of the steady solutions (shown in Figure 4(a)) is the same but the symmetries found for the case of initial conditions (13) are not present; the final profiles are shown in Figure $4(c)$ and are seen to coincide over the hyperbolic part of the domain. Furthermore, the two profiles are locally oscillatory odd functions about $x \approx-1.58$ which is the midpoint of the two elliptic stripes. For general mixed-type flux functions, a sufficient condition for such hyperbolic-elliptic transitions to occur is currently under investigation. Finally we note that for general quadratic nonlinearities there exists a sufficiently large $\epsilon$ so that system decays exponentially and the trivial solution is obtained at large times, analogous to the results of Figure $3(a)$. This is proved using energy estimates in Appendix A. 
$(a)$

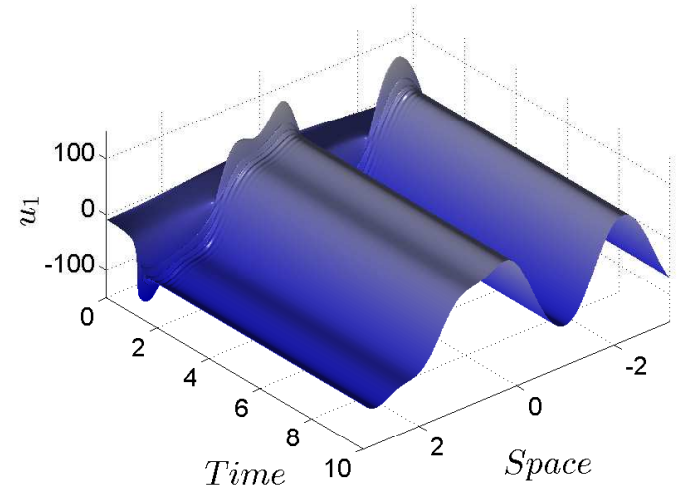

(c)

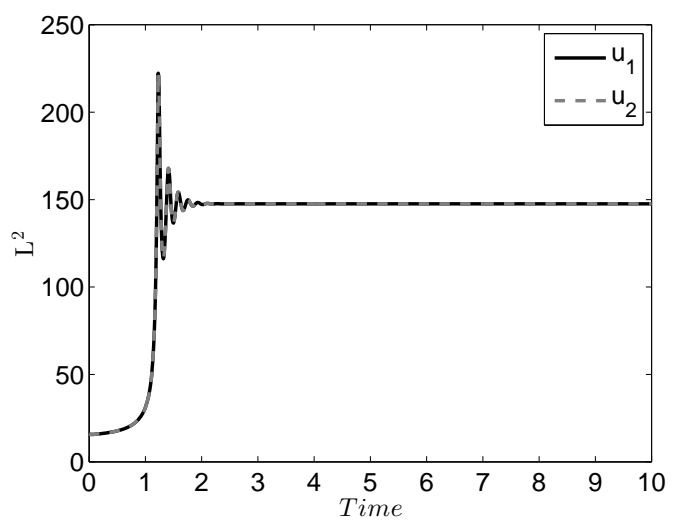

(b)

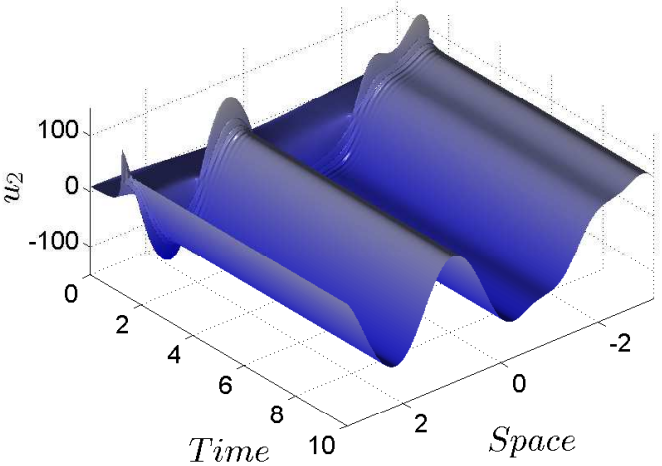

(d)

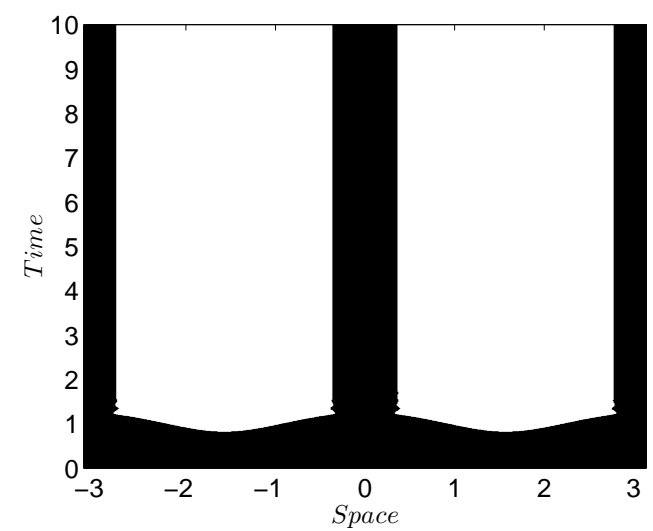

Figure 1. Steady state dynamics exhibited by system (10) with initial conditions $\mathbf{u}_{o}=(9 \cos (x),-9 \cos (x))^{\top}$. The flux function $\mathbf{Q}$ is given by $(12)(a), \rho=4$ and $\epsilon=1$. Panels $(a)$ and $(b)$, the spatiotemporal evolution of $u_{1}$ and $u_{2}$, respectively; panel $(c)$, evolution of the energy; panel $(d)$, the spatiotemporal evolution of the nature of the eigenvalues of $\mathbf{Q}$ : dark-shaded regions correspond to ellipticity (complex eigenvalues) and white-shaded regions indicate hyperbolicity (real eigenvalues).

\subsection{Self-similar solutions for fourth order dissipation $(\rho=4)$ in the limit $\epsilon \rightarrow 0$}

Having established that moderate values of $\epsilon$ can yield non-uniform steady states (see Figure 1), we now concentrate on the behavior of such solutions as $\epsilon$ decreases. This will allow us to probe numerically the vanishing viscosity solutions of the system (10) when $\mathrm{Q}$ is given by $(12)(a)$ and $\rho=4$. We have carried out extensive numerical experiments as $\epsilon$ decreases starting with fixed initial conditions (13). We find that at large times the solutions become steady and have two noteworthy features: (i) Solution maxima (i.e. the $\mathrm{L}^{\infty}$-norms) increase with the relation

$$
\mathrm{L}^{\infty} \sim \epsilon^{-\frac{1}{2}}
$$

as $\epsilon$ decreases, and (ii) the solution develops viscous shock regions of size $\epsilon^{1 / 2}$ that connect fixed states of equal and opposite signs. These properties are supported by 
$(a)$

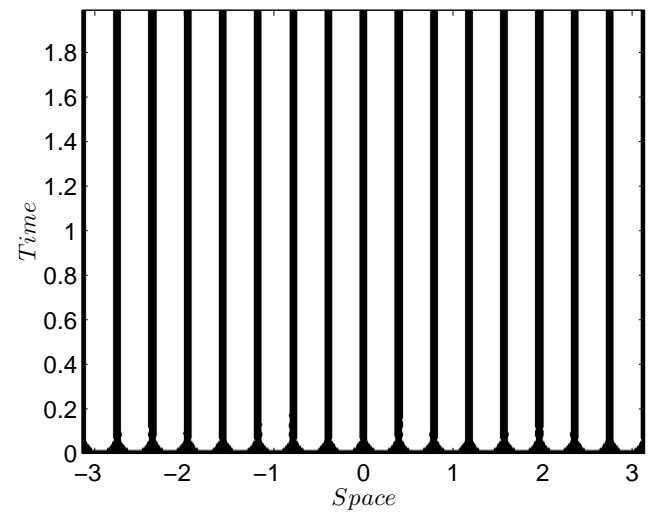

(b)

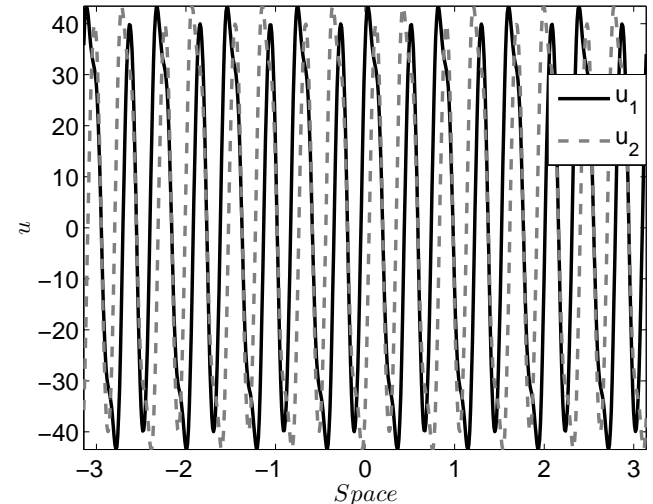

Figure 2. Emergence of 8-modal steady state solutions of system (10) when $\mathbf{Q}$ is given by $(12)(a), \rho=4$ and $\epsilon=0.001$ and starting from $\mathbf{u}_{o}=(9 \cos (8 x),-9 \cos (8 x))^{\top}$. Panel $(a)$, the spatiotemporal evolution of the nature of the eigenvalues: darkshaded regions correspond to complex eigenvalues and white-shaded regions indicate hyperbolicity; panel (b) the final steady state profiles for $u_{1}$ (solid line) and $u_{2}$ (dashed).

$(a)$

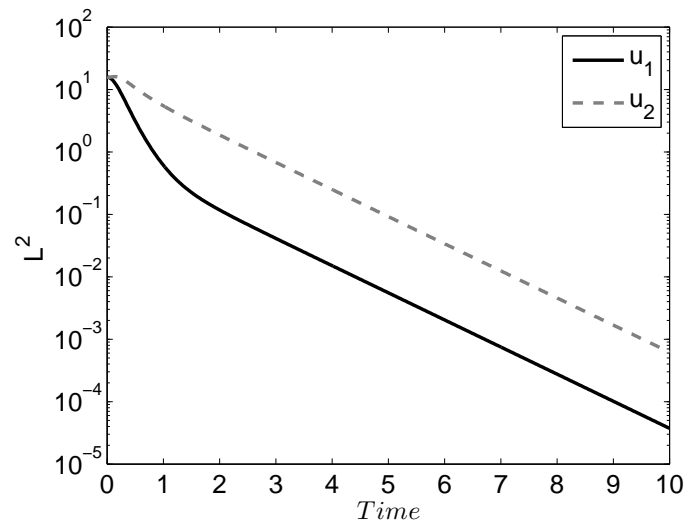

(b)

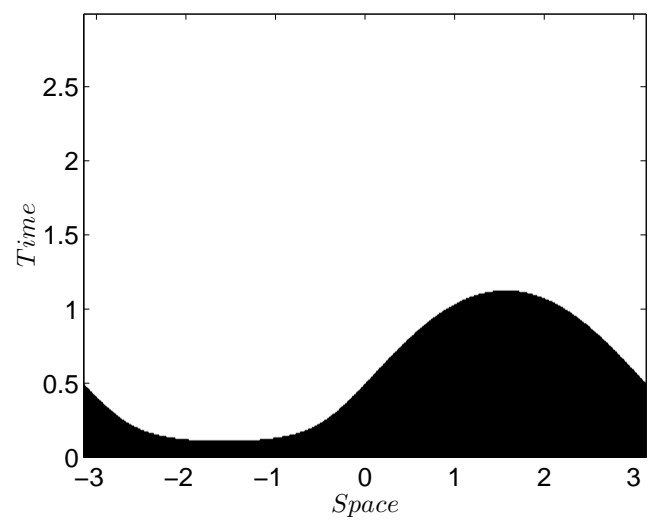

Figure 3. Trivial dynamics exhibited by system (10) when $\mathbf{Q}$ is given by $(12)(b)$, $\rho=4$ and $\epsilon=1$, starting from $\mathbf{u}_{o}=(9 \cos (x),-9 \cos (x))^{\top}$. Panel $(a)$, the evolution of the energy; panel $(b)$, the spatiotemporal evolution of the nature of the eigenvalues (dark - elliptic; white - hyperbolic).

the numerical evidence provided in Figures 5(a,b). Figure 5(a) shows the variation of $\ln \left(\mathrm{L}^{\infty}\right)$ with $\ln (1 / \epsilon)$ as $\epsilon$ approaches zero - note that natural log scales are used. The algebraic behavior (14) is clearly borne by our simulations that superimpose a straight line of slope $1 / 2$ in the log-log plot. The spatial structure of the solutions at $\epsilon=8 \times 10^{-4}$ is depicted in Figure 5(b) which shows the presence of viscous shocks mentioned above. Another interesting feature of the solutions is that they preserve the phase differences imposed initially, i.e. at steady state $u_{1}(x, \cdot)=u_{2}(x+\pi, \cdot)$. 
$(a)$

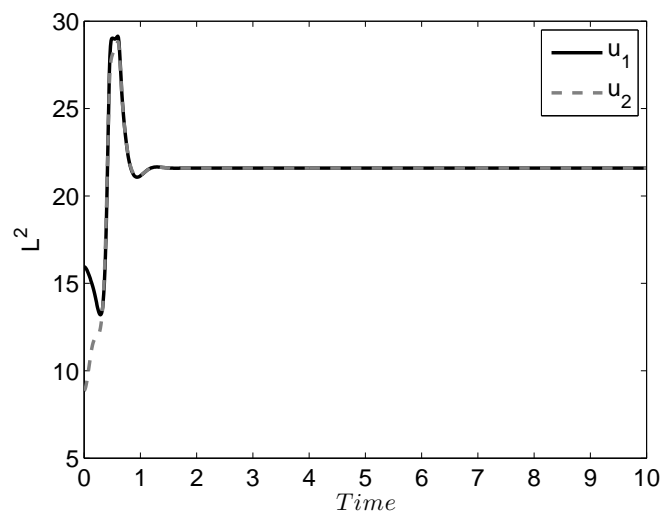

(b)

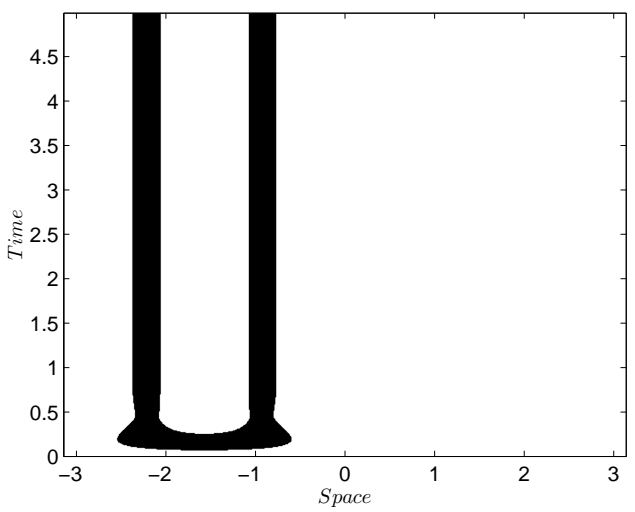

(c)

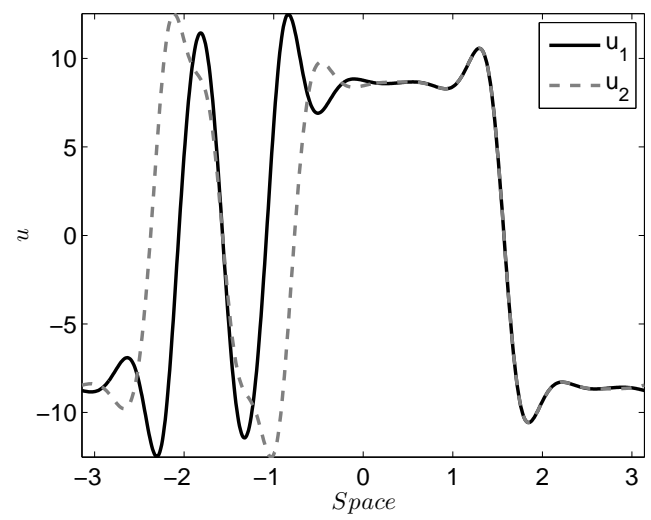

Figure 4. Evolution to steady state for the system (10) starting with hyperbolic initial conditions $\mathbf{u}_{o}=(9 \cos (x), 5 \cos (x))^{\top}$. The flux function $\mathbf{Q}$ is given by $(12)(a), \rho=4$ and $\epsilon=0.01$. Panel $(a)$, the evolution of the energy; panel $(b)$, the spatiotemporal evolution of the nature of the eigenvalues (dark - elliptic; white - hyperbolic); panel (c) final steady-state profiles $u_{1}$ (solid), $u_{2}$ (dashed).

Our numerical experiments strongly suggest that the steady state solutions behave in a self-similar manner as $\epsilon \rightarrow 0$ with universal local behavior in the vicinity of the viscous shocks. To analyze this limit we write

$$
u_{1}(x)=\epsilon^{-1 / 2} U(\xi), \quad u_{2}(x)=\epsilon^{-1 / 2} V(\xi), \quad x-x_{s}=\epsilon^{1 / 2} \xi,
$$

where $x_{s}$ denotes the position of any viscous shock. Note that the scaling for the size of the viscous shock region follows immediately from a balance of nonlinear and viscous terms once the size of the solutions is used from (14). Consequently, in the vicinity of viscous shocks, one can derive the following system of ODEs with the appropriate boundary conditions:

$$
\begin{aligned}
& U_{2} U_{2 \xi}=-U_{1 \xi \xi \xi \xi}, \\
& U_{1} U_{1 \xi}=-U_{2 \xi \xi \xi \xi},
\end{aligned}
$$


(a)

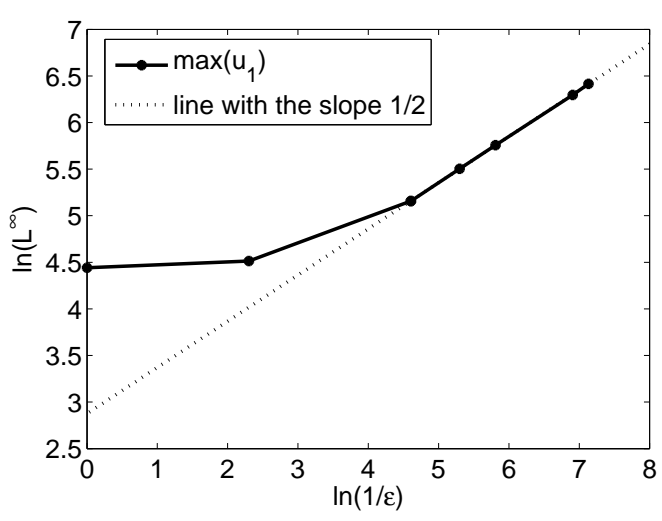

(b)
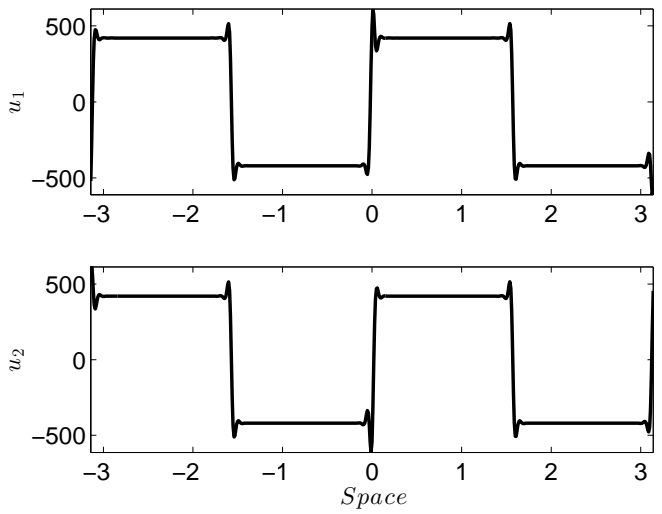

Figure 5. Vanishing viscosity limit for system (10) when $\mathbf{Q}$ is given by $(12)(a), \rho=4$ and $\epsilon$ decreasing. Panel $(a)$ shows the variation of $\ln \left(\mathrm{L}^{\infty}\right)$ with $\ln (1 / \epsilon)$ as $\epsilon$ approaches zero; Panel (b) the final spatial structure of $u_{1}$ (top) and $u_{2}$ (bottom) for $\epsilon=8 \times 10^{-4}$.

$$
U_{1} \rightarrow \pm U_{\infty}, \quad U_{2} \rightarrow \pm U_{\infty} \quad \text { as } \quad \xi \rightarrow \pm \infty, \quad U_{\infty}>0
$$

Using the rescaling $\left(U_{1}, U_{2}\right) \rightarrow U_{\infty}\left(U_{1}, U_{2}\right)$ and $\xi \rightarrow U_{\infty}^{-1 / 3} \xi$ normalizes the equations to the same system (16)-(17) but with the boundary conditions

$$
U_{1} \rightarrow \pm 1, \quad U_{2} \rightarrow \pm 1 \quad \text { as } \quad \xi \rightarrow \pm \infty .
$$

This Riemann problem (with boundary conditions (19)) was solved numerically and compared with the solutions of the PDEs for $\epsilon=8 \times 10^{-4}$ shown in Figure 5(b) (appropriately rescaled to match the ODEs). This was done for the viscous shocks in the vicinity of $x_{s}=0$ and $x_{s}=\pi / 2$, and the results for $u_{1}$ are shown in Figure $6(a)$ and $6(b)$, respectively. Agreement is seen to be excellent. The numerical results provide evidence that as $\epsilon \rightarrow 0$ we do not obtain $\mathrm{L}^{\infty}$ bounds for the solutions.

Finally, we emphasize that the final steady states obtained for different $\epsilon$ (not necessarily small), are not unique but depend crucially on the initial conditions. We have shown solutions supporting four viscous shocks starting with initial conditions (13). However, altering the initial conditions to include more oscillations (but keeping the phase difference), allows us to evolve to steady states with an arbitrary number of elliptic regions (see for example the stripes shown in Figure 2) and consequently an arbitrary number of viscous shocks when in addition $\epsilon$ decreases.

\subsection{Dynamics for lower order dissipation, $\rho<4$ : Finite-time blowup and self-similar solutions}

In this subsection we investigate the effect of different dissipation operators parametrized by $\rho$ as defined in (9). For different values $2 \leq \rho<4$ we solve the initial value problem with initial conditions (13). Both cases corresponding to the flux functions $(12)(a)$ and $(12)(b)$ are considered; we find that when $\epsilon$ is sufficiently large the solutions tend to 
(a)

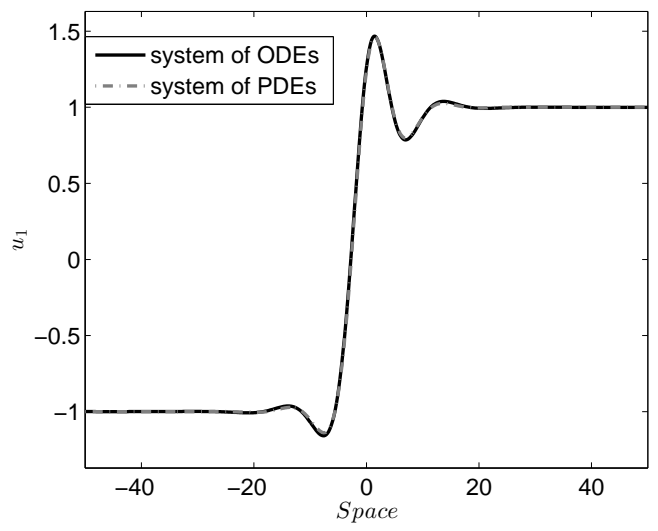

(b)

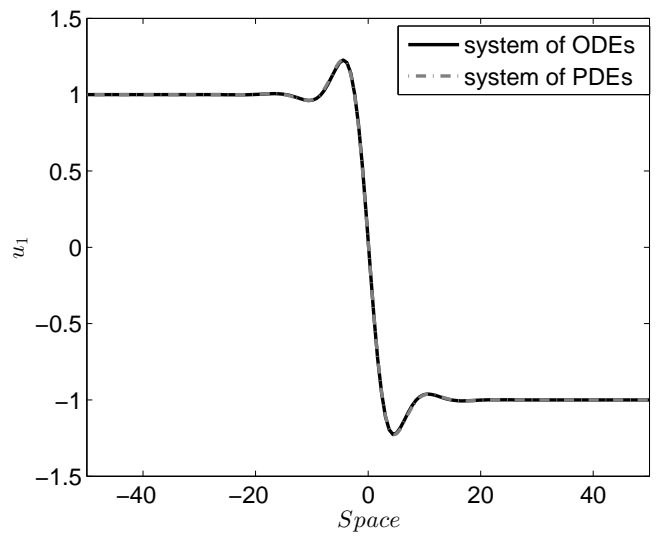

Figure 6. Comparisons of the solutions of system (10) when $\mathbf{Q}$ is given by $(12)(a)$, $\rho=4$ and $\epsilon=8 \times 10^{-4}$ with self similar solutions produced by the ODE system. Panel ( $a$ ) and panel ( $b$ ) depict the viscous shocks of $u_{1}$ in the vicinity of $x_{s}=0$ and $x_{s}=\pi / 2$ respectively.

the trivial state exponentially fast as $t \rightarrow \infty$. On the other hand if $\epsilon$ is sufficiently small, the solutions encounter finite time blowup as we describe in more detail below. A numerically constructed bifurcation diagram in $\epsilon-\rho$ space is given in Figure 7 with panels $(a)$ and $(b)$ corresponding to flux functions $(12)(a)$ and $(12)(b)$, respectively. Note that the depicted boundaries are determined numerically by imposing a uniform criterion of blowup as follows (boundaries between trivial and steady states are much easier to establish computationally): Given a value of $\rho$, the computations proceed with high but fixed resolution in space and time (selected to retain numerical stability), to find the smallest value of $\epsilon$ correct to two significant figures, for which blowup does not occur - these are depicted as dots on the diagram. The results indicate distinct behavior of solutions depending on the type of flux function used. For flux function $(12)(b)$, trivial states give way to blowup irrespective of the value of $\rho$, as can be seen in $7(b)$. In contrast to this, the case of flux function $(12)(a)$ yields more intricate behavior and can support several steady-state windows prior to blowup taking place at smaller $\epsilon$ (all steady states found in this subsection are analogous to those described for $\rho=4$, and possess fixed hyperbolic and elliptic intervals in $x$ - see Figure 1(d) for example). As can be seen from Figure 7(a), when $\rho$ is smaller that approximately 3, trivial steady-states directly give way to solutions blowing up in finite time. However, when $\rho$ is larger than approximately 3 , trivial steady states now give way to non-uniform steady solutions of different type depending on where we are in the $\epsilon-\rho$ space. For instance, when $\rho$ is less than approximately 3.54 a single steady state window is found, labeled I in the figure, while for $\rho$ larger than approximately 3.54 an additional steady state window appears, labeled II in the figure. The boundary between regions I and II shown by the dashed curve, was determined numerically; the solutions attain a jump in their $\mathrm{L}^{2}$-norm when this is crossed. In both flux function cases, our numerical results indicate that the 
curve separating blowup from steady states, has a vertical asymptote as $\rho$ tends to 4 from below and $\epsilon$ tends to zero. The numerical computations near this point become extremely challenging, but the results depicted in Figure $7(a)$ are consistent with those for $\rho=4$ described in subsection 3.2, where we do not find finite-time blowup for non-zero values of $\epsilon$ as small as $8 \times 10^{-4}$.

In what follows we consider blowup solutions in more detail. To illustrate matters we take $\rho=2$ and $\epsilon=1$ in (10) with flux function $(12)(a)$, and solve the problem numerically subject to initial conditions (13) (analogous results hold for flux function $(12)(b))$. Numerical results are depicted in Figure 8. Panel $(a)$ shows the evolution of the $\mathrm{L}^{2}-$ norm of $u_{1}$ (the $\mathrm{L}^{2}-$ norm of $u_{2}$ is identical), and it can be established that a singularity is encountered after a finite time with the norm blowing up. Numerical solutions as $\epsilon$ decreases (also $\rho=2$ and initial conditions as above) have also been carried out and confirm that the singular time is non-zero and tends to a finite value in the limit (results not shown for brevity) - in fact for $\epsilon=10^{-4}$ we find $t_{s} \approx 0.087$. It is possible, therefore, to study the zero viscosity limit over a finite time interval in this case - this is left for future work. The corresponding profiles at the final computed time $t=0.1603$ are given in panel $(b)$, which shows that the solutions become unbounded locally at $x=0$ (and $x=\pi$ ). We also note that the solutions preserve the phase difference imposed initially (i.e. shifting $u_{1}$ spatially by $\pi$ yields $u_{2}$ ). These results provide an example of a parabolic system with second order diffusion that does not possess globally bounded solutions.

The structure of the singular solutions can be analyzed asymptotically for any $\rho$ near the space-time singularity. To achieve this we consider order-of-magnitude estimates of the terms in the PDEs. Supposing that the solution becomes singular at $t=t_{s}, x=x_{s}$, then for $0<t_{s}-t \ll 1$ and $\left|x-x_{s}\right| \ll 1$, balancing terms in (10) yields

$$
\frac{u_{1}}{t_{s}-t} \sim \frac{u_{2}^{2}}{\left|x-x_{s}\right|} \sim \frac{u_{1}}{\left|x-x_{s}\right|^{\rho}}, \quad \frac{u_{2}}{t_{s}-t} \sim \frac{u_{1}^{2}}{\left|x-x_{s}\right|} \sim \frac{u_{2}}{\left|x-x_{s}\right|^{\rho}} .
$$

This in turn provides the scalings $\left|x-x_{s}\right| \sim\left(t_{s}-t\right)^{\frac{1}{\rho}}, u_{1,2} \sim\left(t_{s}-t\right)^{-\left(1-\frac{1}{\rho}\right)}$, and hence we can find self-similar solutions of the form

$$
u_{1,2}=\left(t_{s}-t\right)^{-\left(1-\frac{1}{\rho}\right)} F_{1,2}(\xi), \quad \xi=\frac{x-x_{s}}{\left(t_{s}-t\right)^{\frac{1}{\rho}}} .
$$

Substitution of (21) into the PDEs (10) provides a system of coupled nonlinear ODEs for the scaling functions $F_{1}$ and $F_{2}$ to be solved on $-\infty<\xi<\infty$. For brevity we do not pursue such solutions here, but instead proceed to verify the self-similar ansatz (21) from our numerical solutions. To accomplish this we consider the $\mathrm{L}^{2}$-norms of $u_{1,2}$ and $\partial_{x} u_{1,2}$ which on use of $(21)$ are seen to scale as

$$
\begin{aligned}
& E_{1,2}^{2} \equiv\left\|u_{1,2}(\cdot, t)\right\|_{2}^{2} \sim\left(t_{s}-t\right)^{\left(-2+\frac{3}{\rho}\right)} \\
& E_{1,2 x}^{2} \equiv\left\|\partial_{x} u_{1,2}(\cdot, t)\right\|_{2}^{2} \sim\left(t_{s}-t\right)^{\left(-2+\frac{1}{\rho}\right)} .
\end{aligned}
$$


$(a)$

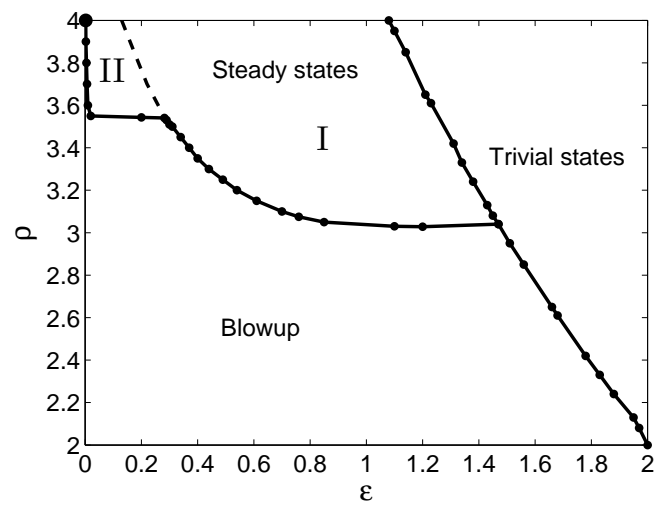

(b)

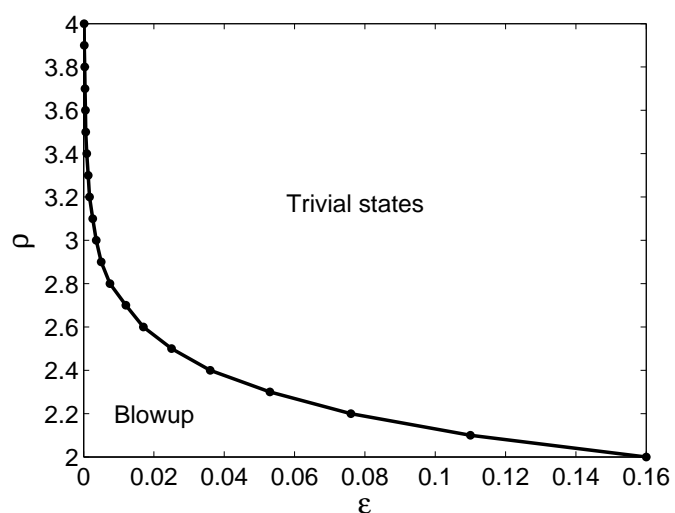

Figure 7. Bifurcation diagram in the $\epsilon-\rho$ space produced by (10) when starting from (13). Panels $(a)$ and $(b)$ corresponding to flux functions (12)(a) and (12)(b), respectively.

Since $2 \leq \rho<4$, the quantities $E_{1,2}$ and $E_{1,2 x}$ blow up as $t \rightarrow t_{s-}$, and from (22) and (23) we obtain

$$
\left(E_{1,2}\right)^{\frac{2}{2 \rho-3}} \sim\left(E_{1,2 x}\right)^{\frac{2}{2 \rho-1}} .
$$

This expression is useful in verifying the self-similar ansatz since it does not require knowledge of the singular time $t_{s}$. In fact, taking logarithms we have

$$
\ln \left(E_{1,2}\right)=\left(\frac{2 \rho-3}{2 \rho-1}\right) \ln \left(E_{1,2 x}\right)+\text { const.. }
$$

Figure $9(a)-(c)$ shows the variation of $\ln \left(E_{1} x\right)$ with $\ln \left(E_{2}\right)$ as the singular time is approached for three values of $\rho=2,2.5,3$. According to the theoretical predictions, the variation should become linear as $t \rightarrow t_{s}$ in all cases, with slopes $1 / 3,1 / 2$ and $3 / 5$, respectively. In each panel we superimpose this prediction and it can be seen that agreement is very good, thus confirming the self-similar structures.

To summarize, this section considered solutions of the coupled parabolic system (10) with homogeneous quadratic flux functions possessing complex eigenvalues. Our numerical results indicate that homogeneous quadratic flux functions (e.g. (6)) play a crucial role in the large time dynamics of the parabolic systems considered here. It is found that the large time solutions are unbounded in the $\infty$-norm as $\epsilon$ tends to zero for all values of $\rho$. If $\rho<4$, solutions blow up in finite time for sufficiently small $\epsilon$, while when $\rho=4$ steady states emerge possessing self-similar local behavior for arbitrarily small $\epsilon$. The mechanism that drives such structures has been identified to hinge on the persistence in time of fixed intervals in $x$ where the solutions produce complex eigenvalues for the flux functions - for example see the stripes shown in Figures $1(d)$ and $4(b)$. Such vertical stripes remain as $\epsilon$ decreases and are the source of the unboundedness of the solutions in the limit. In the following section we generalize the flux functions to include non-homogeneous terms (physically these are advective terms), 
(a)

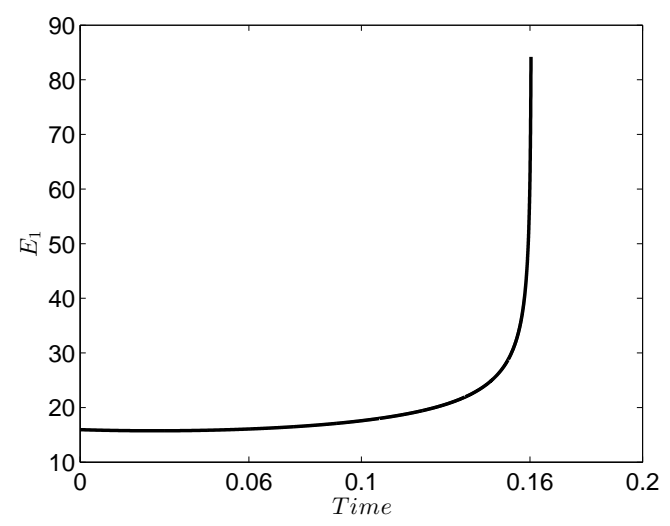

(b)

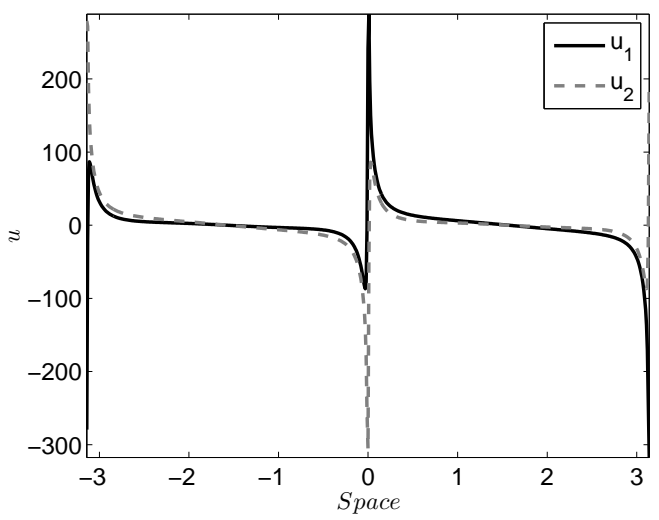

Figure 8. Formation of infinite-slope singularities after finite time for system (10) subject to initial conditions (13). Flux function $\mathbf{Q}$ is given by (12)(a) and $\rho=2, \epsilon=1$. Panel $(a)$ shows the evolution of the $\mathrm{L}^{2}-$ norm of $u_{1}$; panel $(b)$ shows the corresponding profiles at the final computed time $t=0.1603$.

and study vanishing viscosity limits in such cases. The advective terms are found to play a crucial role in the existence of such limits and the boundedness of $\mathrm{L}^{\infty}$-norms.

\section{Vanishing viscosity limits in the presence of linear terms in the flux function, $\zeta \neq 0$}

So far we have studied the dynamical behaviour of generalized parabolic systems which incorporate mixed-type homogeneous quadratic nonlinearities. In this section we investigate such systems but for inhomogeneous flux functions that incorporate advective terms. In particular we consider the system

$$
\frac{\partial \mathbf{u}}{\partial t}+\left(\zeta \mathbf{A}+\nabla_{\mathbf{u}} \mathbf{Q}\right) \frac{\partial \mathbf{u}}{\partial x}=-\epsilon \mathbf{I}\left(\mathcal{H} \circ \partial_{x}\right)^{\rho}[\mathbf{u}],
$$

where

$$
\nabla_{\mathbf{u}} \mathbf{Q}=\left(\begin{array}{cc}
-2 u_{1} & 0 \\
0 & 2 u_{2}
\end{array}\right), \quad \mathbf{A}=\left(\begin{array}{cc}
-0.519 & 0.123 \\
-0.247 & -0.341
\end{array}\right)
$$

As discussed already, the eigenvalues of the flux function are of crucial importance. These are given by

$$
\operatorname{eig}\left(\zeta \mathbf{A}+\nabla_{\mathbf{u}} \mathbf{Q}\right)=\lambda_{1,2}(x, t),
$$

and are complex (i.e. the inviscid system is elliptic) when

$$
\Lambda=\left\{\left[-2\left(u_{1}(x, t)+u_{2}(x, t)\right)-0.519 \zeta+0.341 \zeta\right]^{2}-0.1215 \zeta^{2}\right\}<0 .(29)
$$

Condition (29) is used below in the accurate tracking of elliptic regions in the $x-t$ plane that may be present as the system evolves. System (26) is solved subject to random 
(a)

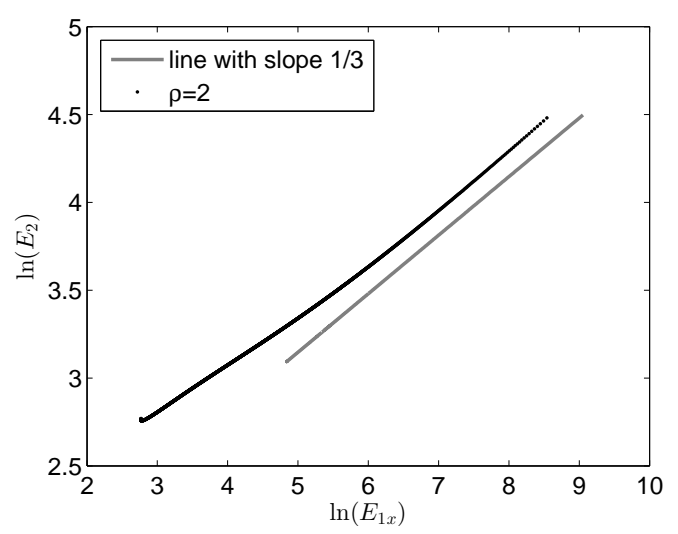

(b)

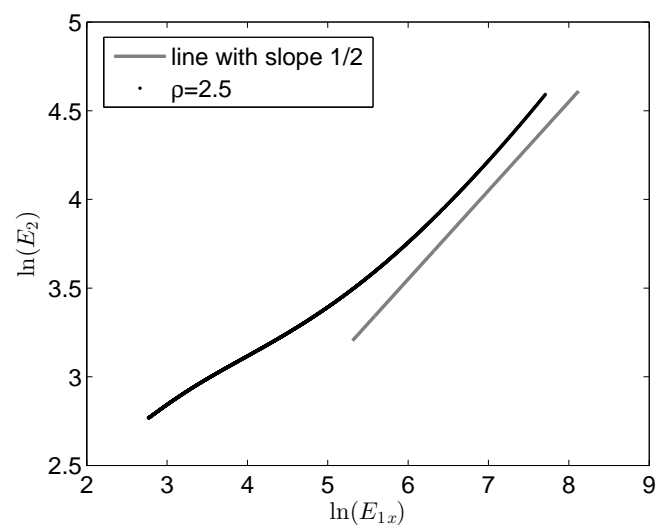

$(c)$

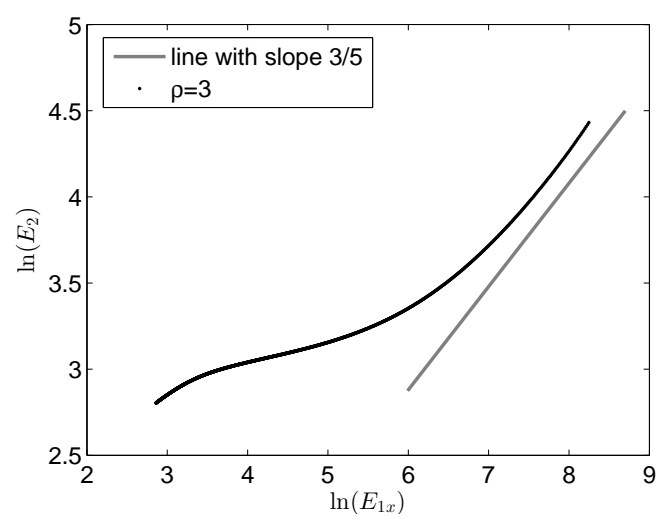

Figure 9. Blow-up diagnostics for $\rho=2($ panel $(a)), \rho=2.5($ panel $(b))$ and $\rho=3$ (panel $(c)$ ); $\epsilon$ is chosen to be inside the blow-up region shown in Figure $7(a)$. The variation of $\ln \left(E_{2}\right)$ with $\ln \left(E_{1 x}\right)$ is depicted as the singular time is approached along with the theoretical prediction (25) for each case.

$2 \pi$-periodic initial conditions with Fourier modes initialized with random amplitudes of size 0.1 or less. The constant matrix $\mathbf{A}$ as well as the flux function, have been chosen to correspond to a specific physical problem as described in [12]. This physical situation is quite generic and is characterized by decoupled nonlinearities and the matrix $\mathbf{A}$ having complex eigenvalues (note that analogous results to those described below have been found in computations with coupled nonlinearities). The latter property implies that the linearized problem (about the state $\mathbf{u}=\mathbf{0}$ ) is short-wave unstable in the absence of dissipation. For finite values of $\epsilon$ and $\rho=4$, numerical experiments in [12] show that the solutions evolve to non-uniform steady-state travelling waves; an example is given in Figure 10 for $\zeta=1, \epsilon=0.1$ and $\rho=4$. We also remark that the speed of propagation decreases as $\zeta$ decreases. (In the results that follow, $\zeta$ is fixed to unity.) Figure 10 $(d)$ depicts the evolution of the hyperbolic-elliptic regions in the $x-t$ plane, which are seen to develop along lines of finite slope to produce inclined stripes; this is in contrast to the vertical stripes found in the absence of linear terms in the flux function (see Figure 
$(a)$

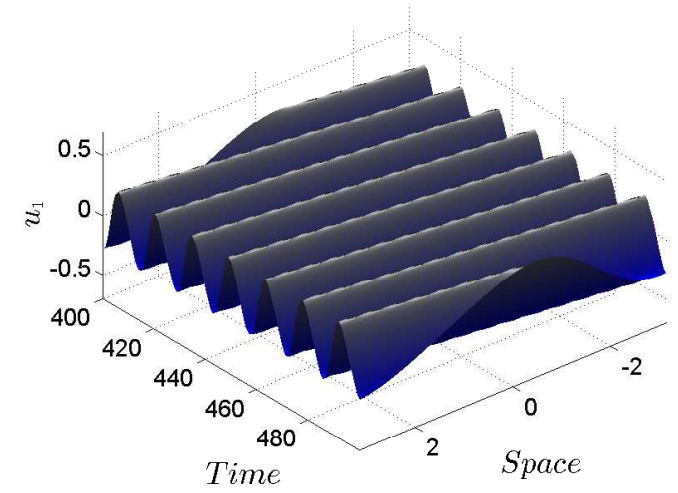

$(c)$

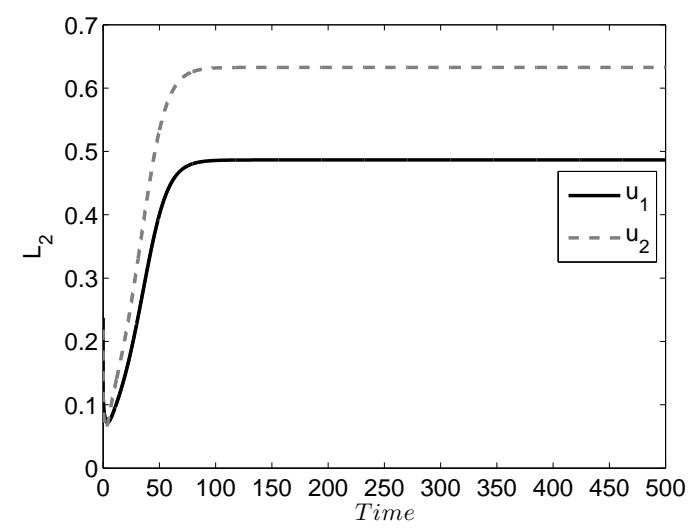

(b)

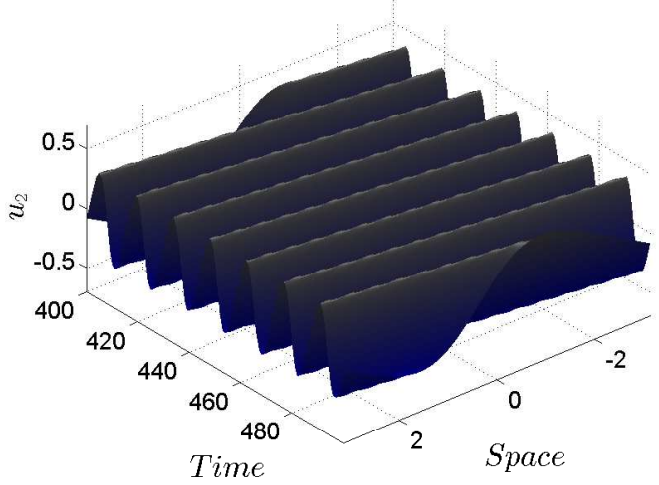

$(d)$

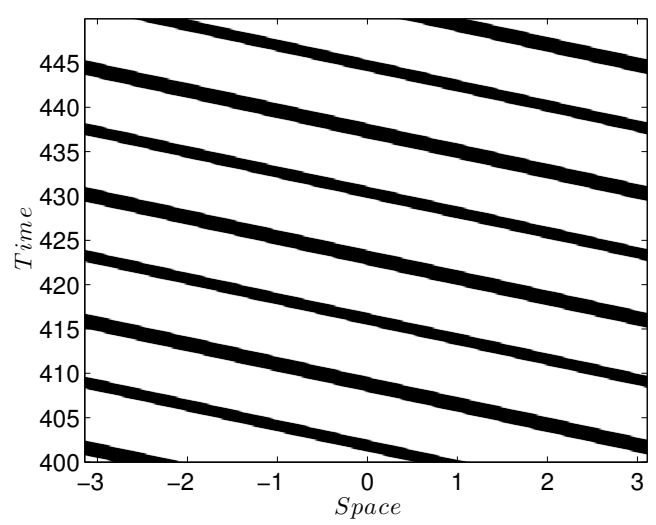

Figure 10. Emergence of steady state traveling waves of system (26) when $\zeta=1$, $\rho=4$ and $\epsilon=0.1$. Panels $(a)$ and $(b)$, show the spatiotemporal evolution of the variables $u_{1}$ and $u_{2}$, respectively; panel $(c)$, evolution of the energy; panel $(d)$, spatiotemporal evolution of the nature of the eigenvalues (dark- complex eigenvalues, white - real eigenvalues.

1). Consequently, hyperbolic-elliptic transitions are the hallmark of the dynamics when linear terms are present. As will be clarified later, this is an essential mechanism in the existence of bounded solutions as $\epsilon$ decreases.

Our main interest is in describing the solutions as $\epsilon$ decreases. Representative results for the solution $u_{1}$ for three small values of $\epsilon=5 \times 10^{-5}, 5 \times 10^{-6}, 4 \times 10^{-7}$, are shown in Figure 11. The solutions are taken after large-time numerical integration starting from the same random initial conditions. The results provide several notable features. As $\epsilon$ decreases additional oscillations appear with the solutions remaining bounded in in the $\mathrm{L}^{\infty}$ norm. Rather than counting the number of oscillations we have found it much more useful to consider the number and size of elliptic regions as indicated in Figure 10(d), for example. This novel way will enable us to understand and quantify the emergence of ever increasing oscillations along the vanishing viscosity route. The spatiotemporal evolution of the elliptic regions for the solutions corresponding to Figure 
11, are given in Figure 12, with panel (a) corresponding to $\epsilon=5 \times 10^{-5}$ and panel (b) to $\epsilon=5 \times 10^{-6}$. The number of elliptic regions increase as $\epsilon$ decreases. We identify the number of elliptic regions by taking a fixed time and counting their number over the whole $2 \pi$ domain. From Figure 12 we find that there are 8 initially (panel $(a)$ ), then increase to 16 (panel $(b)$ ) and further increase to 24 when $\epsilon=4 \times 10^{-7}$ (not shown). Note that in all our computations the number of elliptic regions as $\epsilon$ tends to zero, was found to be always even. At the same time, the intervals in $x$ that support ellipticity, decrease with $\epsilon$. The distribution in space of this increasing set of elliptic regions appears to follow a fractal self-similar structures as we illustrate next.

To produce numerical results that are capable of quantifying fractal structures, it is essential to have highly accurate numerical values for the size and distribution of elliptic regions supported by the solutions $u_{1}, u_{2}$ at a fixed time. The elliptic regions are found over intervals in $x$ where $\Lambda<0$ - see equation (29). We find the edges of all elliptic regions present, by calculating the zeros of the function $\Lambda=0$. All calculations were carried out by using 6th order interpolating polynomials in real space using fine discretization grids of mesh-size $10^{-4}$ (the latter is achieved by spectral interpolation). This in turn implies that the interpolating error is below computer accuracy. In general, then, for a fixed value of $\epsilon$ we find $n$ elliptic regions of size $\chi_{1}, \ldots, \chi_{n}$ - in fact as $\epsilon$ decreases we find that $n$ elliptic regions are supported for a range of $\epsilon$. Numerical evidence for fractal self-similarity is provided in Figure 13. The figure shows the distribution and the actual widths ( $\chi_{i}$ as defined above) over $[-\pi, \pi]$ of the elliptic regions as their number increases (i.e. $\epsilon$ decreases) for the three values of $\epsilon$ represented in Figure 11. The objective is to test whether an enlargement of the distributions for $n=24$ and $n=16$, can produce the structure found in the base case $n=8$. The regions to be compared have been enclosed in the dashed rectangular boxes shown in panel $(a)$. The $n=24$ and $n=16$ results are enlarged by factors of approximately 2.4 and 2 , respectively, and plotted (after a horizontal shift) on top of the unscaled $n=8$ results as shown in panel $(b)$ of Figure 13. Agreement is reasonable and this motivates us to use our computations to estimate the Hausdorff dimension based on the diminishing size and increasing number of the elliptic regions for a wide range of $\epsilon \ll 1$. It is useful to define $\mathcal{X}_{n}=\max _{i=1, \ldots, n}\left\{\chi_{i}\right\}$ which is the largest elliptic interval for a given $n$ (this corresponds to the largest $\epsilon$ that supports $n$ elliptic regions); this in turn allows us to define the Hausdorff dimension of the distribution of elliptic regions

$$
\mathcal{D}=\lim _{n \rightarrow \infty} \frac{\ln (n)}{\ln \left(\frac{1}{\mathcal{X}_{n}}\right)} .
$$

In the numerical computations we calculate $\mathcal{D}_{n}=\frac{\ln (n)}{\ln \left(\frac{1}{\mathcal{X}_{n}}\right)}$ and estimate $\mathcal{D}$ by allowing $n$ to increase (equivalently $\epsilon$ to decrease). Results are presented in Figure 14 for two values of $\rho=4$ and 3.75 corresponding to different dissipation operators, see (26). As $n$ increases the Hausdorff dimension appears to settle down to different values that depend on $\rho$; for $\rho=4$ it is found to be approximately 0.74 while for $\rho=3.75$ we find a value of approximately 0.57 . The decrease with $\rho$ of the Hausdorff dimension, is due 


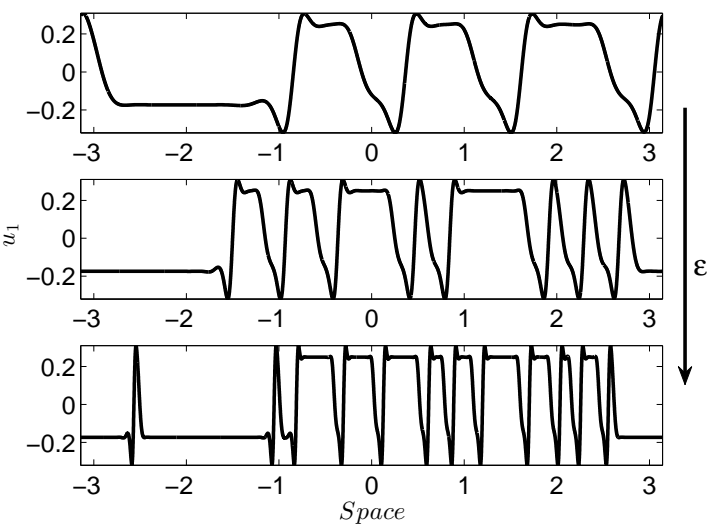

Figure 11. Ever-increasing bounded oscillations in space for system (26) when $\zeta=1$ and $\rho=4$ as $\epsilon$ decreases. From top to bottom, final profiles of $u_{1}$ for three different values of $\epsilon=5 \times 10^{-5}, 5 \times 10^{-6}$ and $4 \times 10^{-7}$.

$(a)$

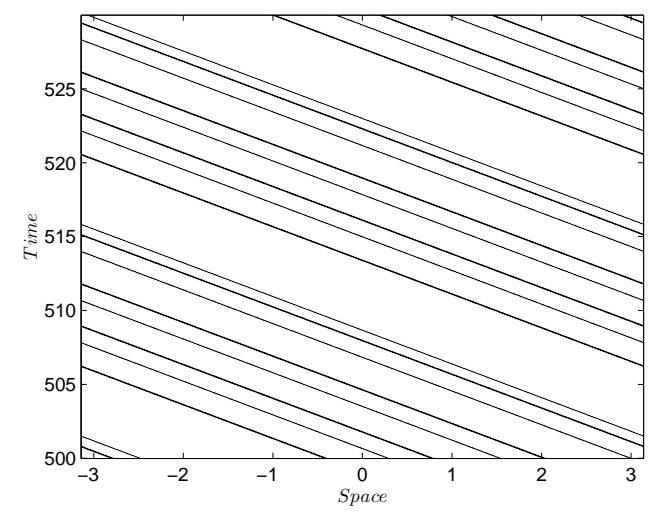

(b)

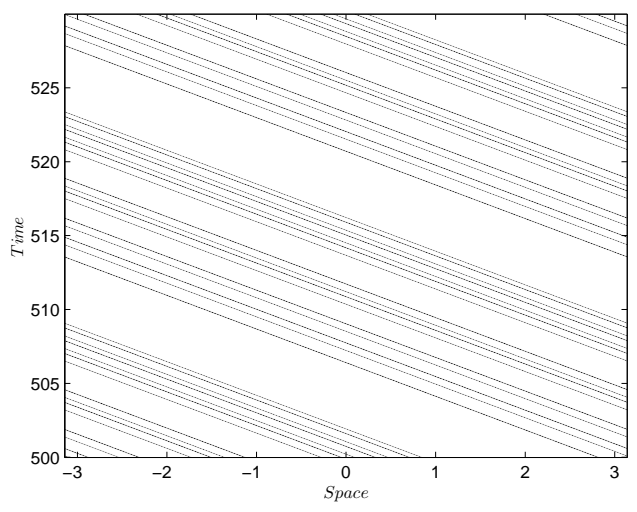

Figure 12. The spatiotemporal evolution of the elliptic regions (black-shaded) of system (26) when $\zeta=1$ and $\rho=4$. Panel (a) corresponds to $\epsilon=5 \times 10^{-5}$ (8 elliptic regions); panel $(b)$ corresponds to $\epsilon=5 \times 10^{-6}$ (16 elliptic regions).

to the sharper gradients appearing in the solutions as the order of the dissipation $\rho$ decreases, leading to smaller elliptic regions. We note that the computations are quite challenging as $\epsilon$ and $\rho$ decrease and require small time-steps and large numbers of modes. The highest number of modes employed in this work was 800 for $\rho=4$ and 1200 for $\rho=3.75$, and these were chosen so that the magnitude of the highest retained mode is at most $10^{-10}$. In the results of Figure 14 we computed as many as 28 elliptic regions so that $\epsilon$ needs to be taken as small as $2.5 \times 10^{-7}$ for $\rho=4$ and $2 \times 10^{-8}$ for $\rho=3.75$.

The numerical experiments described here, provide strong evidence that the $\mathrm{L}^{\infty}$ norm of solutions to (26) remains bounded as the dissipation parameter $\epsilon$ decreases to zero. We conjecture that in the vanishing viscosity limit, solutions exist and become measure-valued. More specifically the solution supports elliptic regions that are confined 
to a Cantor-like set in space with Hausdorff dimension between 0 and 1 that depends on $\rho$, the order of the dissipative term in (26). Consequently, in the limit the system becomes almost everywhere hyperbolic (except on a $\rho$-dependent fractal set with Lebesgue measure zero), and oscillates infinitely many times between two constant states of equal and opposite value so as to preserve the zero spatial mean. Furthermore, the jumps (discontinuities) follow the fractal distribution of the elliptic regions. The connection discovered here between the structure of the regularizing operator and the topology of the elliptic regions (or equivalently the topology of discontinuities) in the zero dissipation limit, provides numerical evidence that may be useful in the classification of measure-valued solutions as posed by DiPerna $[21,31]$.

\subsection{Interpretation of the results on the unscaled domain: Existence of extensive dynamics.}

All numerical results presented previously are based on the scaled $2 \pi$-periodic equation (9). It is interesting to transform the solutions back to unscaled variables so as to obtain solutions of problem (5) on periodic domains of length $L$. In view of the transformation (7), and concentrating on the results of Figure 11 that correspond to fourth order dissipation, we can recover the unscaled solutions by the transformation $x \rightarrow \epsilon^{1 / 3} \xi$, where $\xi \in[0, L]_{\text {per }}$. As $L$ increases we obtain solutions that exhibit extensive dynamics which are strikingly analogous to those of the single Kuramoto-Sivashinsky (KS) equation $([36,37])$. In particular, the solution as well as its derivatives remain bounded as $L$ increases. (This is an important finding regarding the physical problem the weakly nonlinear long-wave description used here is seen to be valid as $L$ increases.) Confirmation of this is provided in Figure 15 which shows the log-log plot of the energy norm of the unscaled solution $u_{1}$ as a function of $L$, i.e. the variation with $L$ of

$$
\mathcal{E}_{1}=\left(\int_{0}^{L} u_{1}^{2} d \xi\right)^{1 / 2}
$$

The results show a linear dependence with $L$ as expected when the dynamics are extensive. Note that analogous results hold for energy norms of the second solution $u_{2}$. We have also confirmed that higher Sobolev norms also grow linearly with $L$, in direct analogy with extensive dynamics behaviour of the Kuramoto-Sivashinsky equation, i.e.

$$
\begin{aligned}
& \eta_{\tau}+\eta \eta_{\xi}+\eta_{\xi \xi}+\eta_{\xi \xi \xi \xi}=0, \\
& \eta(\xi+L, \tau)=\eta(\xi, \tau) .
\end{aligned}
$$

The numerical results presented above exhibit strong connections between the qualitative behaviour of solutions of our system (see equation (5)) with that of the single Kuramoto-Sivashinsky equation. However, the equations have differences in the way in which energy is pumped into the systems by instability mechanisms (they share fourth order dissipation). For the KS equation instability is due to the presence of the linear negative diffusion term (physically arising from inertial effects), whereas in our system (5) energy is generated by the hyperbolic-elliptic transitions that are a direct 
$(a)$

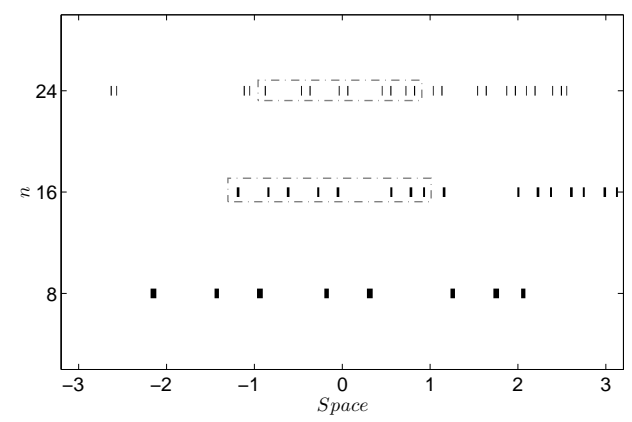

(b)

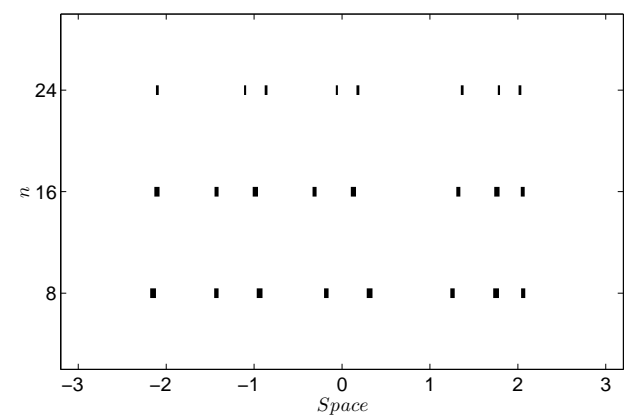

Figure 13. Self-similar behavior of the elliptic regions of system (26) when $\zeta=1$ and $\rho=4$ as $\epsilon$ decreases. Panel $(a)$ shows the distribution and the actual widths of the elliptic regions over $[-\pi, \pi]$ for the three values of $\epsilon=5 \times 10^{-5}$ (8 elliptic regions, bottom), $5 \times 10^{-6}$ (16 regions, middle) and $4 \times 10^{-7}$ (24 regions, top). Panel (b) shows the enlargement of the dashed rectangular boxes shown in panel $(a)$ along with the actual structure of the case of 8 elliptic regions corresponding to $\epsilon=5 \times 10^{-5}$ (bottom panel).

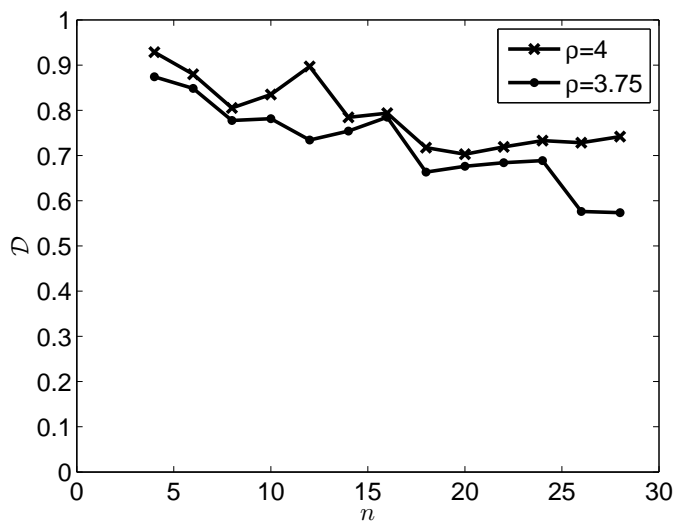

Figure 14. Numerically estimated Hausdorff dimension $(\mathcal{D})$ of the distribution of elliptic regions for two values of $\rho=4$ and 3.75 .

consequence of the nonlinear flux function. More precisely, as $L$ increases the maximum width, $\mathcal{W}_{n}=\mathcal{X}_{n}\left(\epsilon^{-1 / 3}\right)$ say, of the size of the intervals where the flux function is elliptic, is found to remain constant as increasingly more elliptic regions are added. Hence, we surmise that as $L$ increases, $n$ tends to infinity and $\lim _{n \rightarrow \infty} \mathcal{W}_{n}=$ const.. Numerical results suggest that for system (5) when $\mathcal{L}=\mathbf{I} \partial_{\xi}^{4}$, the asymptotic size of the elliptic regions is approximately 1.77 .

\section{Conclusions}

In this paper we studied the vanishing viscosity limits of $2 \times 2$ mixed hyperbolic-elliptic type conservations laws incorporating general quadratic nonlinear flux functions. These 


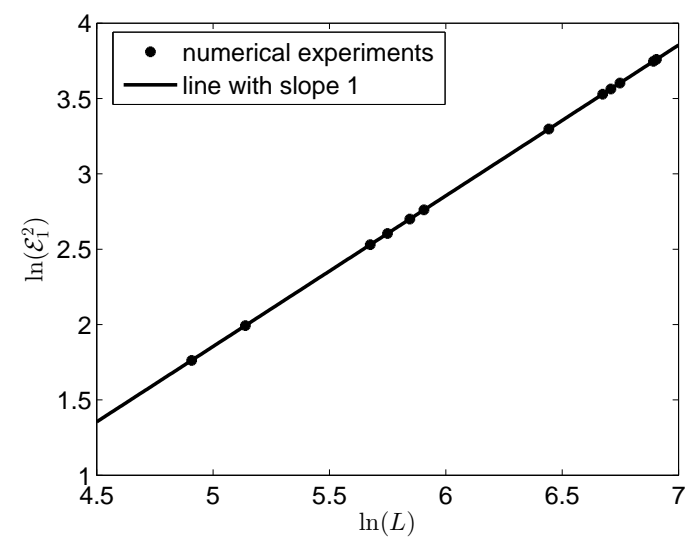

Figure 15. Confirmation of the existence of extensive dynamics for system (5) when $\mathcal{L}=\mathbf{I} \partial_{\xi}^{4}$. The linear dependence of $\ln \left(\mathcal{E}_{1}^{2}\right)$ with the length of the system $L$ is depicted as $L$ increases.

systems are physically motivated and arise in the coupled interfacial dynamics of viscous immiscible multilayer channel flows. The equations contain high order linear dissipation terms (e.g. arising from surface tension), homogeneous quadratic nonlinearities (a result of weak nonlinearity), as well as coupled linear terms. A crucial aspect of the present work is that the flux function is of mixed hyperbolic-elliptic type, i.e. can support real or complex eigenvalues; the only source of instability arises from the presence of such complex eigenvalues. We study numerically the initial value problem on scaled $2 \pi$-periodic domains, and pay particular attention to the vanishing viscosity limits for different dissipation operators that range between second and fourth order. Note that the dissipation parameter $\epsilon$ decreases as the unscaled length of the system increases, as is familiar in scalar equations such as the Kuramoto-Sivashinsky equation. Extensive numerical computations and asymptotic analysis suggest that the existence of bounded vanishing viscosity solutions depends crucially on the structure of the flux function. In particular it is shown that the linear terms play a crucial role in both the dynamics as well as the boundedness of solutions.

In the absence of linear terms (i.e. the flux function is homogeneous) the vanishing viscosity limit does not exist in the $\mathrm{L}^{\infty}$-norm and the solutions become unbounded in a manner that depends on the order of the dissipation term. Fourth order dissipation typically leads to self-similar steady-states that become unbounded as $\epsilon \rightarrow 0$. On the other hand, our numerical experiments indicate that dissipation of order less than four leads to solutions blowing up in finite-time (self-similar structures near the singular time have been constructed and favourably compared to the computations).

If linear terms are present (i.e. the flux function is an inhomogeneous quadratic polynomial), the computations strongly suggest that the solutions exist and are bounded in the $\mathrm{L}^{\infty}$-norm as $\epsilon \rightarrow 0$. In particular the presence of the linear terms introduces spatiotemporally varying hyperbolic-elliptic transitions, which in turn promote highly 
oscillatory but bounded solutions (these are surmised to be measure-valued solutions). We emphasize that as far as we are aware, the classification of measure-valued solutions according to the structure of the regularizing operator is an open problem, see DiPerna $[21,31]$. The present numerical study on spatially periodic domains, facilitates a numerically-based classification in the context of mixed hyperbolic-elliptic systems with quadratic nonlinearities. We observe that the distribution of the oscillations that emerge in the vanishing viscosity limit for high order dissipation (i.e. as large as four), follows a fractal geometry reminiscent of the Cantor set. Furthermore, the fractal dimension is found to depend on the order of the dissipation operator, and decreases as the order of the dissipation decreases (see Figure 14 for example). Finally, when we consider the unscaled problem on $L$-periodic domains, the ever-increasing spatial oscillations can be seen as the emergence of extensive dynamics as $L$ increases. This phenomenon is analogous to the dynamics of the Kuramoto-Sivashinsky equation but in a completely different mathematical context.

Finally we make some comments regarding interesting extensions of this work. The results presented were valid for physical situations where the second order derivative term in (2) was either absent or stabilising. For sufficiently large Reynolds numbers and/or unstably density stratified flows, this term acts as negative diffusion thus providing additional instabilities. In work under way we are considering solutions for general flux functions as the whole operator (including fourth order dissipation and second order negative diffusion) vanishes.

\section{Acknowledgments}

ESP acknowledges the support of a Roth Ph.D. scholarship by the Department of Mathematics, Imperial College London. The work of DTP was partly supported by the EPSRC grant number EP/K041134/.

\section{Appendix A. Proof of large time uniform states for sufficient amount of viscosity and small initial conditions}

Here we use energy estimates to prove that under certain conditions the system (10) with a general quadratic flux function $Q$ given by (6) and $\rho=4$, the solutions decay exponentially in time to trivial uniform states. The result holds for sufficiently large viscosity $\epsilon$ and sufficiently small initial conditions as shown below.

Consider the following set of nonlinear parabolic PDEs

$$
\begin{aligned}
& u_{1 t}+2 a_{1} u_{1} u_{1 x}+2 b_{1}\left(u_{2} u_{1 x}+u_{1} u_{2 x}\right)+2 c_{1} u_{2} u_{2 x}+\epsilon u_{1 x x x x}=0, \\
& u_{2 t}+2 a_{2} u_{2} u_{2 x}+2 b_{2}\left(u_{2} u_{1 x}+u_{1} u_{2 x}\right)+2 c_{2} u_{1} u_{1 x}+\epsilon u_{2 x x x x}=0 .
\end{aligned}
$$

By multiplying (A.1) and (A.2) by $u_{1}$ and $u_{2}$, respectively, and integrating over the 
periodic domain $\Omega=[-\pi, \pi]$ with respect to $x$, we obtain

$$
\begin{aligned}
& \int_{\Omega} u_{1 t} u_{1} d x+\int_{\Omega} 2 a_{1} u_{1}^{2} u_{1 x} d x+\int_{\Omega} 2 b_{1}\left(u_{1}^{2} u_{2 x}+u_{1} u_{2} u_{1 x}\right) d x \\
& +\int_{\Omega} 2 c_{1} u_{2} u_{2 x} u_{1} d x+\epsilon \int_{\Omega} u_{1 x x x x} u_{1} d x=0, \\
& \int_{\Omega} u_{2 t} u_{2} d x+\int_{\Omega} 2 a_{2} u_{2}^{2} u_{2 x} d x+\int_{\Omega} 2 b_{1}\left(u_{2}^{2} u_{1 x}+u_{1} u_{2} u_{2 x}\right) d x \\
& +\int_{\Omega} 2 c_{2} u_{1} u_{1 x} u_{2} d x+\epsilon \int_{\Omega} u_{2 x x x x} u_{2} d x=0 .
\end{aligned}
$$

Integrating by parts and using periodicity yields:

$$
\begin{aligned}
& \frac{1}{2} \frac{d}{d t} \int_{\Omega} u_{1}^{2} d x-\int_{\Omega} c_{1} u_{2}^{2} u_{1 x} d x+\int_{\Omega} b_{1} u_{1}^{2} u_{2 x} d x+\epsilon \int_{\Omega} u_{1 x x}^{2} d x=0, \\
& \frac{1}{2} \frac{d}{d t} \int_{\Omega} u_{2}^{2} d x-\int_{\Omega} c_{2} u_{1}^{2} u_{2 x} d x+\int_{\Omega} b_{2} u_{2}^{2} u_{1 x} d x+\epsilon \int_{\Omega} u_{2 x x}^{2} d x=0 .
\end{aligned}
$$

Adding equations (A.5) and (A.6) and using the Cauchy-Schwartz inequality, we obtain

$$
\begin{aligned}
& \frac{1}{2} \frac{d}{d t}\left(\left\|u_{1}\right\|_{2}^{2}+\left\|u_{2}\right\|_{2}^{2}\right) \leq\left(\left|b_{1}\right|+\left|c_{2}\right|\right)\left\|u_{1}^{2}\right\|_{2}\left\|u_{2 x}\right\|_{2} \\
& +\left(\left|b_{2}\right|+\left|c_{1}\right|\right)\left\|u_{2}^{2}\right\|_{2}\left\|u_{1 x}\right\|_{2}-\epsilon\left(\left\|u_{1 x x}\right\|_{2}^{2}+\epsilon\left\|u_{2 x x}\right\|_{2}^{2}\right) .
\end{aligned}
$$

Use of Young's inequality in (A.7) allows us to express it as

$$
\begin{aligned}
& \frac{1}{2} \frac{d}{d t}\left(\left\|u_{1}\right\|_{2}^{2}+\left\|u_{2}\right\|_{2}^{2}\right) \leq\left(\left|b_{1}\right|+\left|c_{2}\right|\right)\left(\delta_{1}\left\|u_{1}^{2}\right\|_{2}^{2}+\frac{\delta_{1}^{-1}}{4}\left\|u_{2 x}\right\|_{2}^{2}\right) \\
& +\left(\left|b_{2}\right|+\left|c_{1}\right|\right)\left(\delta_{1}\left\|u_{2}^{2}\right\|_{2}^{2}+\frac{\delta_{1}^{-1}}{4}\left\|u_{1 x}\right\|_{2}^{2}\right)-\epsilon\left(\left\|u_{1 x x}\right\|_{2}^{2}+\left\|u_{2 x x}\right\|_{2}^{2}\right),
\end{aligned}
$$

where $\delta_{1}$ is a positive constant.

The next step is to use the Gagliardo-Nirenberg interpolation inequalities (see [38]) in order to find an upper bound for the higher norm $\left\|u^{2}\right\|_{2}^{2}=\|u\|_{4}^{4}$, appearing in (A.8). The relevant inequalities in one space read: Let $\Omega \subset \mathbb{R}, 1 \leq q \leq p \leq \infty$ and $1 \leq r \leq \infty$. Then there exists a constant $C$ such that

$$
\|u\|_{p} \leq C\|u\|_{q}^{1-a}\|u\|_{W^{1, r}}^{a},
$$

where $a=\left(\frac{1}{q}-\frac{1}{p}\right) /\left(\frac{1}{q}-\frac{1}{r}+1\right)(0 \leq a \leq 1)$ and $\|u\|_{W^{1, r}}=\|u\|_{r}+\left\|u_{x}\right\|_{r}$. Hence, taking $p=4$ and $q=r=2$ yields

$$
\begin{aligned}
& \|u\|_{4}^{4} \leq\left(C\|u\|_{2}^{\frac{3}{4}}\|u\|_{W^{1,2}}^{\frac{1}{4}}\right)^{4}=C^{4}\|u\|_{2}^{3}\|u\|_{W^{1,2}} \\
& \leq C^{4}\left(\delta_{2}\|u\|_{2}^{6}+\frac{\delta_{2}^{-1}}{4}\|u\|_{W^{1,2}}^{2}\right),
\end{aligned}
$$


where the last bound follows from Young's inequality. Applying the Poincaré inequality now gives

$$
\|u\|_{4}^{4} \leq C^{4}\left(\delta_{2}\|u\|_{2}^{6}+\frac{\delta_{2}^{-1}}{2}\left\|u_{x x}\right\|_{2}^{2}\right)
$$

where $\delta_{2}$ is a positive constant.

Now, given inequality (A.11) and choosing $\delta_{1}=\delta_{2}=\frac{1}{2}$, allows us to express (A.8) as follows

$$
\begin{aligned}
& \frac{1}{2} \frac{d}{d t}\left(\left\|u_{1}\right\|_{2}^{2}+\left\|u_{2}\right\|_{2}^{2}\right) \leq\left(\left|b_{1}\right|+\left|c_{2}\right|\right)\left(\frac{C^{4}}{4}\left\|u_{1}\right\|_{2}^{6}+\frac{C^{4}}{2}\left\|u_{1 x x}\right\|_{2}^{2}+\frac{1}{2}\left\|u_{2 x}\right\|_{2}^{2}\right) \\
& +\left(\left|b_{2}\right|+\left|c_{1}\right|\right)\left(\frac{C^{4}}{4}\left\|u_{2}\right\|_{2}^{6}+\frac{C^{4}}{2}\left\|u_{2 x x}\right\|_{2}^{2}+\frac{1}{2}\left\|u_{1 x}\right\|_{2}^{2}\right)-\epsilon\left(\left\|u_{1 x x}\right\|_{2}^{2}+\left\|u_{2 x x}\right\|_{2}^{2}\right) .
\end{aligned}
$$

If $\epsilon$ satisfies the condition

$$
2 \epsilon>\max \left\{C^{4}\left(\left|b_{1}\right|+\left|c_{2}\right|\right)+\left|b_{2}\right|+\left|c_{1}\right|, C^{4}\left(\left|b_{2}\right|+\left|c_{1}\right|\right)+\left|b_{1}\right|+\left|c_{2}\right|\right\},
$$

then the Poincaré inequality readily yields

$\frac{d}{d t}\left(\left\|u_{1}\right\|_{2}^{2}+\left\|u_{2}\right\|_{2}^{2}\right) \leq A_{1}\left(\left\|u_{1}\right\|_{2}^{2}\right)^{3}+A_{2}\left(\left\|u_{2}\right\|_{2}^{2}\right)^{3}-A_{3}\left\|u_{1}\right\|_{2}^{2}-A_{4}\left\|u_{2}\right\|_{2}^{2}$

$\leq \max \left\{A_{1}, A_{2}\right\}\left(\left\|u_{1}\right\|_{2}^{2}+\left\|u_{2}\right\|_{2}^{2}\right)^{3}-\min \left\{A_{3}, A_{4}\right\}\left(\left\|u_{1}\right\|_{2}^{2}+\left\|u_{2}\right\|_{2}^{2}\right)$,

where $A_{1}=\frac{C^{4}\left(\left|b_{2}\right|+\left|c_{1}\right|\right)}{2}, A_{2}=\frac{C^{4}\left(\left|b_{1}\right|+\left|c_{2}\right|\right)}{2}, A_{3}=2 \epsilon-\left(C^{4}\left(\left|b_{1}\right|+\left|c_{2}\right|\right)+\left|b_{2}\right|+\left|c_{1}\right|\right)>0$ and $A_{4}=2 \epsilon-\left(C^{4}\left(\left|b_{2}\right|+\left|c_{1}\right|\right)+\left|b_{1}\right|+\left|c_{2}\right|\right)>0$. Finally, by defining $y:=\left\|u_{1}\right\|_{2}^{2}+\left\|u_{2}\right\|_{2}^{2}$, $A:=\max \left\{A_{1}, A_{2}\right\}$ and $B:=\min \left\{A_{3}, A_{4}\right\}$, inequality (A.14) becomes

$$
\frac{d}{d t} y \leq A y^{3}-B y \text {. }
$$

As a result, given that initial conditions for $y$ are below the threshold $\beta=\sqrt{\frac{B}{A}},(y<\beta)$, then due to Gronwall's lemma inequality (A.15) yields

$$
y \leq \frac{\beta}{\sqrt{1+K e^{(2 B t)}}},
$$

where $K>0$. Therefore, inequality (A.16) implies that if (A.13) holds and the sum of the energies of the initial conditions is less than $\beta$, then the solutions of system (A.1)-(A.2) decay exponentially to zero $(y \rightarrow 0)$, as time goes to infinity $(t \rightarrow \infty)$.

\section{References}

[1] Milewski P. et al 2004 Nonlinear stability of two-layer flows Commun. Math. Sci 2 427-442.

[2] Chumakova L. et al 2009 Shear instability for stratified hydrostatic flows Comm. Pure Appl. Math. 62 183-197.

[3] Cole J.D. and Cook L.P. 1986 Transonic Aerodynamics (Elsevier Science Publishers B.V).

[4] Kogan M.N. 1961 On magnetohydrodynamic flows of mixed type J. Appl. Math. Mech. 25 132-137.

[5] Bürger R. et al 2002 Model equations and instability regions for the sedimentation of polydisperse suspensions of spheres Z. Angew. Math. Mech. 82 699-722. 
[6] Slemrod M. 1983 Admissibility criteria for propagating phase boundaries in a van der Waals fluid Arch. Rat. Mech. Anal 81 301-315.

[7] Bell J.B. et al 1986 Conservation laws of mixed type describing three-phase flow in porous media SIAM J. on Appl. Math. 46 1000-1017.

[8] Joseph D.D. et al 1985 Hyperbolicity and change of type in the flow of viscoelastic fluids Arch. Rat. Mech. Anal. 87 213-251.

[9] Fit A.D. 1989 The numerical and analytical solution of ill-posed systems of conservation laws Appl. Math. Modelling 13 618-631.

[10] Krasny R. 1986 A study of singularity formation in a vortex sheet by the point-vortex approximation J. Fluid Mech. 167 65-93.

[11] Keyfitz B.L.et al 2001 Proc. of the Int. Conf. on Multiphase flow Mathematical properties of nonhyperbolic models for incompressible two-phase flow (New Orleans) May 27- June 1 (CD-ROM).

[12] Papaefthymiou E.S. et al 2013 Nonlinear interfacial dynamics in stratified multilayer channel flows J. Fluid Mech. 734 114-143.

[13] Serre D. 2000 Systems of conservation Laws. Vol. I. Hyperbolicity, Entropies, Shock Waves (Cambridge University Press).

[14] Glimm J. 1965 Solutions in the large for nonlinear hyperbolic systems of equations Comm. Pure Appl. Math. 18 697-715.

[15] Lax P.D. 1973 Hyperbolic systems of conservation laws and the mathematical theory of shock waves (Society for Industrial and Applied Mathematics).

[16] Glimm J. and Lax P.D. 1970 Decay of solutions of systems of nonlinear hyperbolic conservation laws (American Mathematical Society).

[17] Baiti P. and Jenssen H.k. 2001 Blowup in $\mathbf{L}^{\infty}$ for a class of genuinely nonlinear hyperbolic systems of conservation laws Discrete Contin. Dyn. S. 7 837-853.

[18] Bressan A. 2011 Open questions in the theory of one dimensional hyperbolic conservation laws (Springer US).

[19] Bianchini S. and Bressan A. 2005 Vanishing viscosity solutions of nonlinear hyperbolic systems Ann. of Math. 161 223-342.

[20] Majda A. and Pego R.L. 1985 Stable viscosity matrices for systems of conservation laws J. Differ. Equations 56 229-262.

[21] DiPerna R.J. 1985 Measure-valued solutions to conservation laws Arch. Rat. Mech. Anal. 88 223-270.

[22] Bertozzi A.L. et al 1999 Undercompressive shock in thin film flows Phys. D 134 431464.

[23] Engelberg S. 1999 An analytical proof of the linear stability of the viscous shock profile of the Burgers equation with fourth-order viscosity SIAM J. on Appl. Math. 4 927-936.

[24] Eyink G.et al 2008 Dissipative anomalies in singular Euler flows Phys. D 237 (14-17) 1956-1968.

[25] Chueh K.N. et al 1977 Positively invariant regions for systems of nonlinear diffusion equations Indiana Univ. Math. J. 26 373-392.

[26] DiPerna R.J. 1983 Convergence of the viscosity method for isentropic gas dynamics Commun. Math. Phys. 91 1-30.

[27] DiPerna R.J. and Majda A. 1987 Oscillations and concentrations in weak solutions of the incompressible fluid equations Commun. Math. Phys. 108 667-689.

[28] Peters G.R. and Canic S. 2000 On the oscillatory solutions in hyperbolic conservation laws Nonlinear Anal. Real World Appl. 1 287-314.

[29] Frid H. and Liu I-S. 1995 Oscillation waves in Riemann problems inside elliptic regions for conservation laws of mixed type $Z$. angew. Math. Phys. 46 913-931.

[30] Berres S. et al 2009 Numerical approximation of oscillatory solutions of hyperbolic- 
elliptic systems of conservation laws by multiresolution schemes Adv. Appl. Math. Mech. 1 581-614.

[31] DiPerna R.J. 1986 Proc. of the Int. Congr. of Mathematicians Compactness of solutions to nonlienar PDE (Berkeley, California, USA) 1057-1063.

[32] Benney D.J. 1966 Long waves on liquid films J. Math. Phys. 45 150-155.

[33] Schaeffer D.G. and Shearer M. 1987 The classification of $2 \times 2$ systems of non-strictly hyperbolic conservation laws, with application to oil recovery Comm. Pure Appl. Math. 40 141-178.

[34] Tseluiko D. and Papageorgiou D.T. 2006 A global attracting set for nonlocal Kuramoto-Sivashinsky equations arising in interfacial electrohydrodynamics Euro J. Appl. Math. 17 677-703.

[35] Hoff D. and Smoller J. 1985 Solutions in the large for certain nonlinear parabolic systems Ann. Inst. Henri Poincaré 2 213-235.

[36] Wittenberg W.R. and Holmes P. 1999 Scale and space localization in the KuramotoSivashinsky equation Chaos 9 452-465.

[37] Otto F. 2009 Optimal bounds on the Kuramoto-Sivashinsky equation J. Funct. Anal. $2572188-2245$.

[38] Brezis H. 2011 Functional analysis, Sobolev spaces and partial differential equations (Springer). 